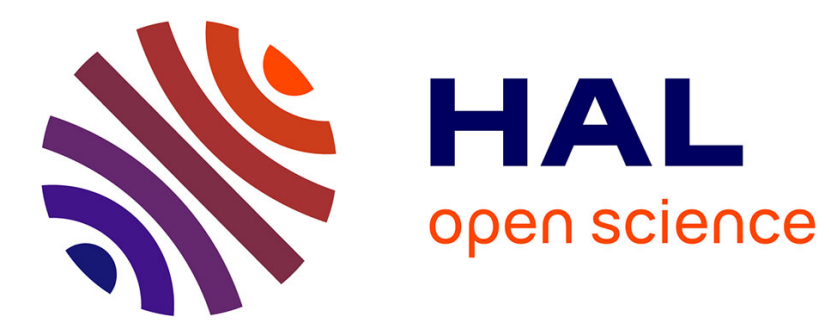

\title{
On 2D Constrained Discrete Rigid Transformations
}

\author{
Phuc Ngo, Yukiko Kenmochi, Nicolas Passat, Hugues Talbot
}

\section{To cite this version:}

Phuc Ngo, Yukiko Kenmochi, Nicolas Passat, Hugues Talbot. On 2D Constrained Discrete Rigid Transformations. Annals of Mathematics and Artificial Intelligence, 2015, 10.1007/s10472-014-9406$\mathrm{x}$. hal-00838184v1

\section{HAL Id: hal-00838184 https://hal.science/hal-00838184v1}

Submitted on 25 Jun 2013 (v1), last revised 16 Nov 2015 (v2)

HAL is a multi-disciplinary open access archive for the deposit and dissemination of scientific research documents, whether they are published or not. The documents may come from teaching and research institutions in France or abroad, or from public or private research centers.
L'archive ouverte pluridisciplinaire HAL, est destinée au dépôt et à la diffusion de documents scientifiques de niveau recherche, publiés ou non, émanant des établissements d'enseignement et de recherche français ou étrangers, des laboratoires publics ou privés. 


\title{
On 2D Constrained Discrete Rigid Transformations
}

\author{
Phuc Ngo ${ }^{1}$, Yukiko Kenmochi ${ }^{1}$, Nicolas Passat ${ }^{2}$, and Hugues \\ Talbot $^{1}$ \\ ${ }^{1}$ Université Paris-Est, LIGM, UPEMLV-ESIEE-CNRS, France \\ ${ }^{2}$ Université de Reims Champagne-Ardenne, CReSTIC, EA 3804, \\ France
}

June 24, 2013

\begin{abstract}
Rigid transformations are involved in a wide range of digital image processing applications. In such a context, they are generally considered as continuous processes, followed by a digitization of the results. Recently, rigid transformations on $\mathbb{Z}^{2}$ have been alternatively formulated as a fully discrete process. Following this paradigm, we investigate -from a combinatorial point of view- the effects of pixel-invariance constraints on such transformations. In particular we describe the impact of these constraints on both the combinatorial structure of the transformation space and the algorithm leading to its generation.

Keywords: Rigid transformation, discrete geometry, combinatorial structure, image processing, pixel-invariance constraints
\end{abstract}

\section{Introduction}

Rigid transformations are frequently involved in applications of computer vision and image processing (e.g., motion tracking [10, 27], image registration [15, 28] or pattern recognition $[4,8])$. In such applications, images are generally digital, and thus defined on finite sets of points in the Eulerian space $\mathbb{Z}^{n}$. However, rigid transformations applied on such digital images are usually performed on the Euclidean space $\left(\mathbb{R}^{n}\right)$. Their results then need to be followed by a subsequent digitization process to finally produce transformed images in $\mathbb{Z}^{n}$.

In a recent work [17], we have proposed to alternatively study rigid transformations ${ }^{1}$ on $\mathbb{Z}^{2}$ as a fully discrete process, similarly to previous contributions related, e.g., to rotations $[3,14,19,20,22,23]$ or quasi-affine transformations

\footnotetext{
${ }^{1}$ In fact, rigid transformations are composed of reflections, rotations and translations, while combinations of only translations and rotations are called proper rigid transformations. In
} 
[6]. In this context, two main questions were considered: ( $i)$ How many rigid transformations can be defined on a finite subspace of $\mathbb{Z}^{2}$ ? (ii) How to generate all these transformations? The difficulty of these questions derives from the infinite number of rigid transformations in $\mathbb{R}^{2}$. Recently, some combinatorial studies were devoted to $2 \mathrm{D}$ pattern matching under different classes of transformations such as rotations, scaling, affine and projective transformations. In particular, some discretization techniques were developed by Hundt et al. $[11,12,13]$. Inspired by these works, we provided in [17] some combinatorial and algorithmic answers to the above two questions, and then contributed to the state of the art in this research area $[2,11,12,13,26]$.

More precisely, in [17], a combinatorial structure, namely a discrete rigid transformation graph (or DRT graph), was introduced to model the parameter space of $2 \mathrm{D}$ rigid transformations. This DRT graph describes all the possible rigid transformations on a digital image. We showed that there exist in the order of $N^{9}$ such transformations, if $N \times N$ is the number of pixels in the image. In addition, the DRT graph explicitly models the "topological links" between such digital transformations, and thus allows the incremental construction of discrete rigid transformations via elementary image modifications. The DRT graph can be used in a local fashion, e.g., in pattern-based strategies, as proposed in [18] for analysing the topological invariance of digital images under arbitrary rigid transformations. Beyond the theoretical aspects of the DRT graph, its highorder polynomial complexity makes it difficult to generate the whole graph for large images, and to use it directly in imaging applications such as registration or warping $[1,9,21,28]$.

To reduce the complexity of this graph, we propose to provide spatial constraints in order to guide the computation of such transformations. Indeed these constraints introduce prior knowledge that contribute to reducing the search space. In this article -that is an extended and improved version of the conference paper [16] - we investigate such constrained search paradigms from the combinatorial and algorithmic points of view. We focus in particular on the effects of geometric constraints on discrete rigid transformations, via the analysis of the DRT graph. More precisely, we investigate pixel-invariance constraints, which consist of enforcing the correspondence between points in an initial subspace of $\mathbb{Z}^{2}$ and points (or more generally regions) in a transformed space.

This article is organised as follows. Section 2 briefly introduces some basic notions of rigid transformations on digital images. Section 3 describes pixelinvariance constraints in the associated parameter space of rigid transformations. In Section 4, we develop an algorithmic process for generating a combinatorial structure modeling all the discrete rigid transformations and their relationships under given constraints. Complexity analyses of the proposed algorithm and the induced structure are described in Section 5. A concluding discussion is finally proposed in Section 6 .

this article, as in [17], we focus on the latter, since any reflection can be easily obtained from rotations. By abuse of language, we will continue to refer to proper rigid transformations as rigid transformations. 


\section{Background notions}

\subsection{Digital images and digital rigid transformations}

In a $2 \mathrm{D}$ continuous space, an image can be defined as a function $\mathcal{I}: \mathbb{R}^{2} \rightarrow$ $\mathbb{V}$, where $\mathbb{V}$ is a given value space. In computer imaging, such images are represented as discrete functions obtained through a sampling process, and then called digital images. In general, the sampling process relies on partitioning $\mathbb{R}^{2}$ into Voronoi cells induced by a square grid structure. It associates almost every point in $\mathbb{R}^{2}$ to a unit grid square (namely, a pixel), and equivalently to a point in $\mathbb{Z}^{2}$. Such a sampling process, also referred as digitization, is often carried out by the following function

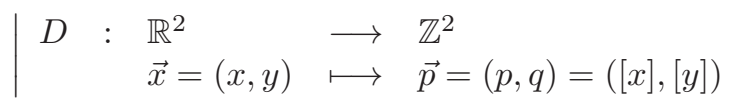

where [.] is a rounding operator. Consequently, a digital image associated to $\mathcal{I}$ can be formalized as $I: \mathbb{Z}^{2} \rightarrow \mathbb{V}$. In other words, we have $I=\mathcal{I}_{\mid \mathbb{Z}^{2}}$, and for each $\vec{p} \in \mathbb{Z}^{2}$, the value $I(\vec{p})$ models the value of $\mathcal{I}$ on the associated pixel $\vec{p}+\left[-\frac{1}{2}, \frac{1}{2}\right]^{2}$, namely the Voronoi cell of $\mathbb{R}^{2}$ induced by $\mathbb{Z}^{2}$ around $\vec{p}$.

A $2 \mathrm{D}$ rigid transformation is defined as a rotation followed by a translation. In the continuous framework, such a transformation can be formally expressed as a bijective function $\mathcal{T}: \mathbb{R}^{2} \rightarrow \mathbb{R}^{2}$ such that for any $\vec{x}=(x, y) \in \mathbb{R}^{2}$, the transformed point $\mathcal{T}(\vec{x})$ has the form

$$
\mathcal{T}(\vec{x})=\left(\begin{array}{rr}
\cos \theta & -\sin \theta \\
\sin \theta & \cos \theta
\end{array}\right)\left(\begin{array}{l}
x \\
y
\end{array}\right)+\left(\begin{array}{l}
a \\
b
\end{array}\right)
$$

where the parameters $a, b \in \mathbb{R}$ represent the translation, while $\theta \in[0,2 \pi[$ is the rotation angle. In particular, such a transformation is unambiguously modeled by the triplet of parameters $(a, b, \theta)$, and will be often denoted by $\mathcal{T}_{a b \theta}$. When applied to an image $\mathcal{I}: \mathbb{R}^{2} \rightarrow \mathbb{V}$, it provides a new transformed image $\mathcal{I} \circ \mathcal{T}$ : $\mathbb{R}^{2} \rightarrow \mathbb{V}$.

It is not possible to apply directly $\mathcal{T}$ to a digital image $I: \mathbb{Z}^{2} \rightarrow \mathbb{V}$, since there is no guarantee that $\mathcal{T}(\vec{x}) \in \mathbb{Z}^{2}$ for $\vec{x} \in \mathbb{Z}^{2}$. In the discrete framework, the handling of digital rigid transformations then requires to define a function $T_{a b \theta}: \mathbb{Z}^{2} \rightarrow \mathbb{Z}^{2}$, which is a discrete analogue of $\mathcal{T}_{a b \theta}$. Following the digitization paradigm $D$ proposed above, a digital rigid transformation $T$ associated to $\mathcal{T}$ can be conveniently performed by setting $T=D \circ \mathcal{T}$, as illustrated in the following diagram.

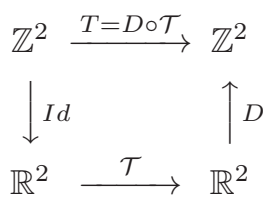

The function $T: \mathbb{Z}^{2} \rightarrow \mathbb{Z}^{2}$ is then explicitly defined for $\vec{p}=(p, q) \in \mathbb{Z}^{2}$ by

$$
T(\vec{p})=D \circ \mathcal{T}(\vec{p})=\left(\begin{array}{l}
{[p \cos \theta-q \sin \theta+a]} \\
{[p \sin \theta+q \cos \theta+b]}
\end{array}\right)
$$




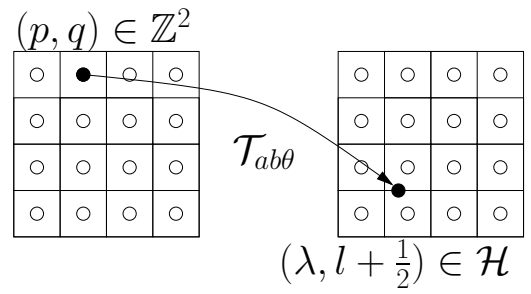

(a)

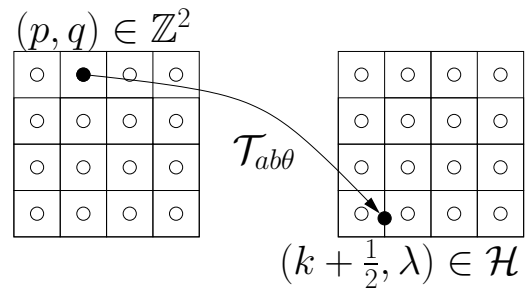

(b)

Figure 1: Examples of critical transformations $\mathcal{T}_{a b \theta}$, that map at least one integer-coordinate point onto a "horizontal" (a) or "vertical" (b) half-grid point. The integer-coordinate points in $\mathbb{Z}^{2}$ are depicted by dots, while the half-grid points are depicted by lines.

In general, this function is not bijective. However, by setting $T^{-1}: \mathbb{Z}^{2} \rightarrow \mathbb{Z}^{2}$ as $T^{-1}=D \circ \mathcal{T}^{-1}$, it becomes possible to define the digital transformed image $I \circ T^{-1}: \mathbb{Z}^{2} \rightarrow \mathbb{V}$ with respect to $T$. In the sequel of this article, we focus on such digital rigid transformations. From this point on -for the sake of readability and without loss of correctness- we will note $T$ instead of $T^{-1}$, due to the bijectivity of $\mathcal{T}$ and $\mathcal{T}^{-1}$.

From a theoretical point of view, the above notions (images, rigid transformations) are defined on $\mathbb{Z}^{2}$ and $\mathbb{R}^{2}$. Practically, our purpose is however to study rigid transformations on images of finite size. Under this hypothesis, only some digital rigid transformations are relevant, namely those that actually have an effect on such finite images. From this point on, we focus on this finite case, and we assume that the digital images are defined on subsets of $\mathbb{Z}^{2}$ of size $N \times N$. Without loss of generality, a digital image $I$ is then written as $I: \mathbb{S} \rightarrow \mathbb{V}$ for $\mathbb{S}=\llbracket 0, N \rrbracket^{2} \subset \mathbb{Z}^{2}$.

\subsection{Discontinuities of digital rigid transformations}

In $\mathbb{R}^{2}$, any rigid transformation $\mathcal{T}_{a b \theta}$ is a continuous function (see Equation (2)). However, this notion of continuity is lost once the function is digitized. Indeed, due to the digitization process involved in the definition of digital rigid transformations (see Equation (4)), the parameter space $(a, b, \theta)$ of rigid transformations is divided into $3 \mathrm{D}$ open cells, in each of which the function $(a, b, \theta) \mapsto T_{a b \theta}=$ $D \circ \mathcal{T}_{a b \theta}$ is constant. In particular, these $3 \mathrm{D}$ open cells are separated by $2 \mathrm{D}$ closed cells corresponding to rigid transformations that map at least one integer coordinate point onto a half-grid point (see Fig. 1). Such transformations, which lead to discontinuities within the parameter space, are called critical transformations.

Definition 1 (Critical transformation [17]) Let $(a, b, \theta) \in \mathbb{R}^{2} \times[0,2 \pi[$, and $\mathcal{T}_{a b \theta}: \mathbb{R}^{2} \rightarrow \mathbb{R}^{2}$ be its associated rigid transformation. We say that $\mathcal{T}_{a b \theta}$ is a critical transformation if there exists $\vec{p} \in \mathbb{Z}^{2}$ such that $\mathcal{T}_{\text {ab } \theta}(\vec{p}) \in \mathcal{H}$, where $\mathcal{H}$ is 


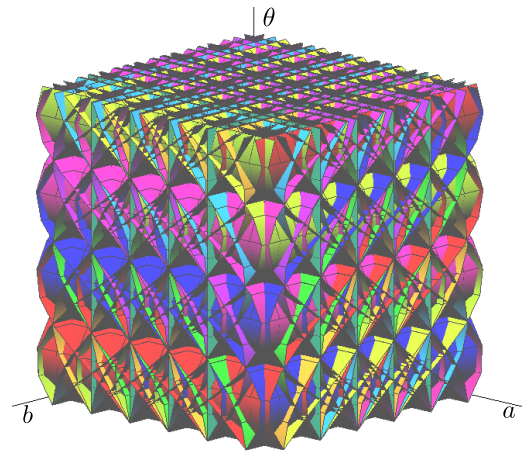

(a)

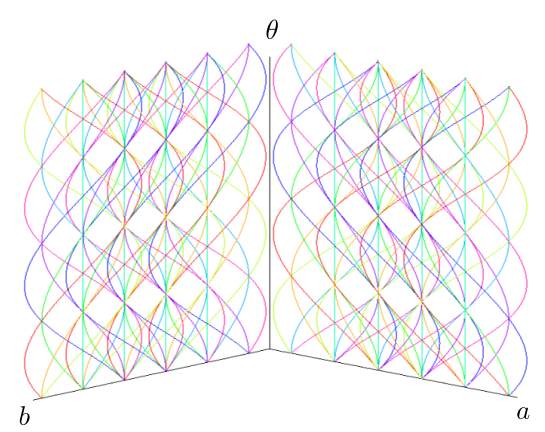

(b)

Figure 2: (a) Tipping surfaces in the 3D parameter space $(a, b, \theta)$, and (b) their cross-sections, namely tipping curves, in the 2D planes $(a, \theta)$ and $(b, \theta)$.

the half-grid defined by

$$
\mathcal{H}=\left[\mathbb{R} \times\left(\mathbb{Z}+\frac{1}{2}\right)\right] \cup\left[\left(\mathbb{Z}+\frac{1}{2}\right) \times \mathbb{R}\right]
$$

Note that the half-grid $\mathcal{H}$ corresponds to the boundaries of the Voronoi cells of $\mathbb{R}^{2}$ induced by $\mathbb{Z}^{2}$.

More precisely, for each $\vec{p}=(p, q) \in \mathbb{Z}^{2}$ that is mapped onto a half-grid point which can be either horizontal $\left(k+\frac{1}{2}, \lambda\right) \in \mathcal{H}$ or vertical $\left(\lambda, l+\frac{1}{2}\right) \in \mathcal{H}$, we have one critical transformation, denoted either $\Phi_{p q k}$ or $\Psi_{p q l}$, defined by the set of $(a, b, \theta)$ that satisfies the following formula

$$
\begin{aligned}
& \Phi_{p q k}: \mathbb{R} \times[0,2 \pi[\longrightarrow \mathbb{R} \\
& (b, \theta) \quad \longmapsto a=\phi_{p q k}(\theta)=k+\frac{1}{2}+q \sin \theta-p \cos \theta \\
& \Psi_{p q l} \quad: \quad \mathbb{R} \times[0,2 \pi[\longrightarrow \mathbb{R} \\
& (a, \theta) \quad \longmapsto \quad b=\psi_{p q l}(\theta)=l+\frac{1}{2}-p \sin \theta-q \cos \theta
\end{aligned}
$$

The $2 \mathrm{D}$ surfaces $\Phi_{p q k}$ (resp. $\left.\Psi_{p q l}\right)$ defined in the parameter space $(a, b, \theta)$ are called tipping surfaces [17]. Their respective cross-section $\phi_{p q k}\left(\right.$ resp. $\left.\psi_{p q l}\right)$ on the 2D plane $(a, \theta)$ (resp. $(b, \theta))$ are called tipping curves. These tipping surfaces/curves, which correspond to the discontinuities of the digital rigid transformations, expressed in the parameter space $(a, b, \theta)$, are illustrated in Fig. 2. It is important to remark that the tipping surfaces $\Phi_{p q k}$ and $\Psi_{p q l}$ can be straightforwardly recovered by extruding the tipping curves $\phi_{p q k}$ and $\psi_{p q l}$, respectively.

\subsection{Partition of the parameter space and DRT graph}

As a result of the discontinuity of digital rigid transformations induced by the digitization process (Equation (1)), it is possible that some distinct rigid transformations (Equation (2)) be mapped onto a same digital rigid transformation 


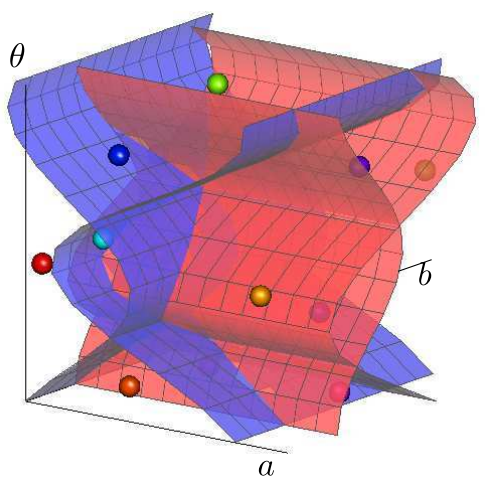

(a)

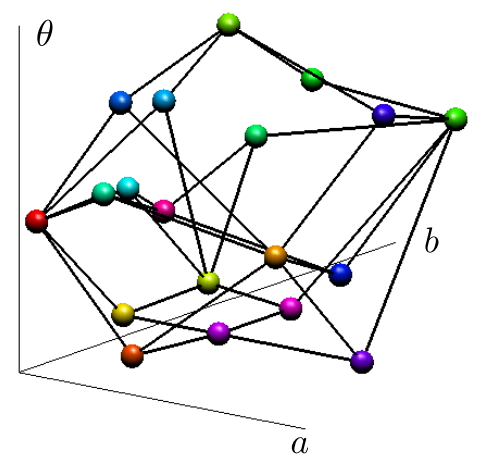

(b)

Figure 3: (a) The parameter space of rigid transformations subdivided by four tipping surfaces, and (b) the associated DRT graph.

(Equation (4)). This leads to considering equivalence classes between transformations, which are defined by the following relation

$$
\left(\mathcal{T}_{a b \theta} \sim \mathcal{T}_{a^{\prime} b^{\prime} \theta^{\prime}}\right) \Longleftrightarrow\left(T_{a b \theta}=T_{a^{\prime} b^{\prime} \theta^{\prime}}\right)
$$

It has to be noticed that this equivalence relation is only defined for non-critical rigid transformations. As stated above, it is possible to identify a rigid transformation with its triplet of parameters $(a, b, \theta)$. In this context, the equivalence classes of transformations, called discrete rigid transformations ${ }^{2}$ (DRTs), can be modeled by $3 \mathrm{D}$ open cells in this parameter space, whose boundaries are 2D tipping surfaces defined above (see Fig. 2(a)). In other words, the parameter space $(a, b, \theta)$ of rigid transformations is partitioned into disjoint sets of noncritical transformations, each of which is associated to exactly one DRT, and bounded by the surfaces modeling critical transformations.

We have shown in [17] that the subdivision of this parameter space could be modeled by using a dual combinatorial structure, that maps each 3D cell (i.e., each DRT) onto a 0D point and each 2D tipping-surface segment (linked to a critical transformation) onto a $1 \mathrm{D}$ edge. The resulting structure is called a DRT graph (see Fig. 3).

Definition 2 (DRT graph [17]) Given a set of tipping surfaces, $\Phi_{p q k}$ and $\Psi_{p q l}$, the graph $G=(V, E)$ associated to DRTs induced by those $\Phi_{p q k}$ and $\Psi_{p q l}$ is defined in the following way:

- each vertex $v \in V$ models a 3D open cell associated to a DRT;

\footnotetext{
${ }^{2}$ Contrarily to the terminology frequently used in the literature, the term digital refers here to the digitization process $D$ defined in Equation (4), while the term discrete refers to the combinatorial structure induced by this operator $D$.
} 
- each labelled edge $e=(u, w, f) \in E$ (where $f$ is either $\Psi_{p q k}$ or $\Phi_{p q l}$ ) models the tipping surface $f$ between two adjacent vertices $v, w \in V$.

This graph $G$ is called a DRT graph.

In [17], we have proved that the space complexity of the DRT graph for any set $\mathbb{S}$ of size $N \times N$ is polynomial. An exact computation algorithm is proposed to build this graph in linear time with respect to the size of the graph.

Property 3 ([17]) The DRT graph associated to a digital image of size $N \times N$ has a space complexity of $\mathcal{O}\left(N^{9}\right)$.

The DRT graph models a kind of "neighbouring" relationship between DRTs. Indeed, by associating a resulting digital transformed image to each 3D open cell/DRT, the existence of a 2D surface between two cells indicates that the associated transformed images differ in exactly one pixel among the $N^{2}$ ones. More precisely, let us consider an edge $e=(v, w, f) \in E$ between two distinct vertices $v, w \in V$. The function $f$ (that is either equal to $\Psi_{p q k}$ or $\Phi_{p q l}$ ) indicates that exactly one point $\vec{p}=(p, q) \in \mathbb{S}$ will cross the half-grid line at coordinates $(x, y) \in \mathbb{R}^{2}$, with either $x=k+\frac{1}{2}$ or $y=l+\frac{1}{2}(k, l \in \llbracket 0, N \rrbracket)$. Practically, let $I_{v}$ and $I_{w}$ be the transformed images corresponding to the vertices $v$ and $w$ respectively. Let $\vec{q}$ be the point with coordinates either $(k, \lambda)$ or $(\lambda, l)$ with respect to $f$. The value of $\vec{q}$ at the vertex $v$ is defined by $I_{v}(\vec{q})=I(\vec{p})$ where $I: \mathbb{S} \rightarrow \mathbb{V}$ is the original image. After the elementary change along edge $e$, we obtain a new transformed image $I_{w}$ by simply setting the pixel value at $\vec{q}$ to $I_{w}(\vec{q})=I(\vec{p}+\vec{\delta})$ where $\vec{\delta}=( \pm 1,0)$ or $(0, \pm 1)$ with respect to $f$. In this way, one can generate all the transformed images of $I$ by incrementally and exhaustively scanning the associated DRT graph. This property, exemplified in Fig. 4, was used in [18] for verifying the topologically invariance of digital images under rigid transformations.

\section{Constraints and feasible rigid transformation sets}

The DRT graph is highly complex in space and time, which make its practical construction and handling challenging for large images. In the sequel, we investigate how the use of constraints may reduce these complexities. More precisely, we focus on pixel-invariance constraints which consist of enforcing correspondence between points in the initial and transformed image. In particular, we expect these constraints to reduce the size of the parameter space partition, in terms of the number of 3D cells, and therefore the size of the associated DRT graph. 


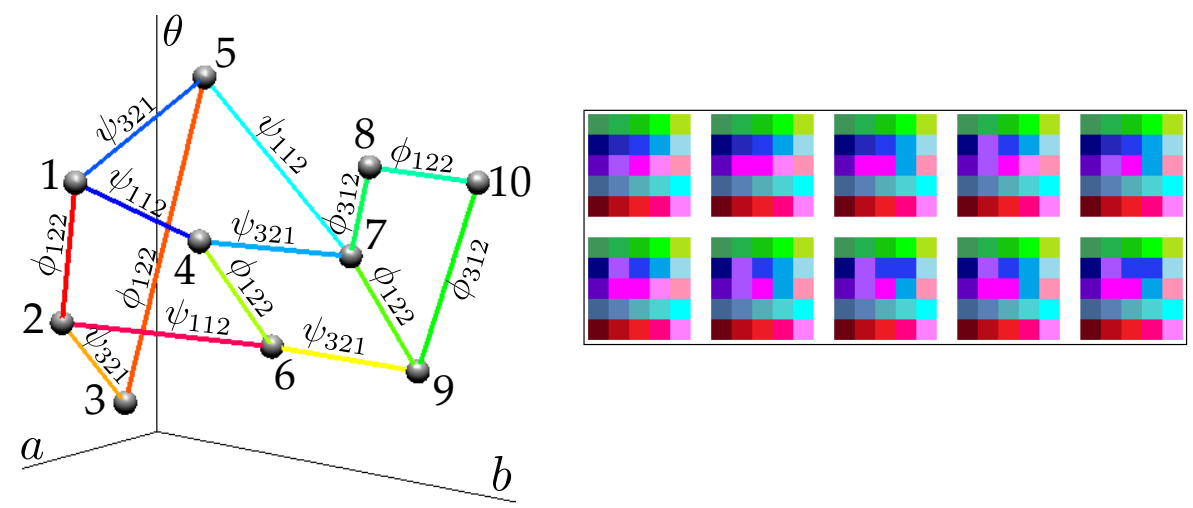

Figure 4: Left: a part of a DRT graph in which a vertex/DRT represents a digital transformed image and an edge $e=(v, w, f)$ between two vertices $v$ and $w$ indicates that one pixel value is different between the associated transformed images $I_{v}$ and $I_{w}$ (see text). Right: the transformed images associated to the vertices of the DRT graph (in left). The images from upper-left to bottom-right correspond to the vertices ordered from 1 to 10 in the graph.

\subsection{Pixel-invariance constraints and interpretation in the parameter space}

In the context of rigid transformations in $\mathbb{R}^{2}$, enforcing the correspondence between two points $\vec{p}$ and $\vec{p}^{\prime}$ in the initial and in the transformed space respectively, leads to restricting the number of authorised transformations. More precisely, from Equation (2) we obtain, for a given pair of corresponding points, two equations representing $2 \mathrm{D}$ trigonometric surfaces, that intersect to provide a 1D trigonometric curve, which models this (affine) space of rotations (see Fig. 5(a)).

A unique constraint then leads to an infinite space of transformations, that we call feasible transformations. In order to obtain a finite space of feasible transformations, we then need two (distinct) constraints, i.e., two pairs of corresponding points $\left(\vec{p}, \vec{p}^{\prime}\right)$ and $\left(\vec{q}, \vec{q}^{\prime}\right)$. If these pairs are well chosen, i.e., they satisfy the isometric properties of rigid transformations $\left(\|\vec{p}-\vec{p}\|_{2}=\left\|\vec{q}-\vec{q}^{\prime}\right\|_{2}\right)$, then the space of feasible transformations is restricted to a unique transformation (see Fig. 5(b)) that corresponds to the intersection of the two $1 \mathrm{D}$ curves induced by these constraints. Otherwise, the space of feasible transformations is empty. More generally, setting the correspondence between $k \geq 2$ distinct couples of points $\left(\vec{p}_{i}, \vec{p}_{i}^{\prime}\right)$, for $i \in \llbracket 1, k \rrbracket$, restricts the authorised transformations to at most a single feasible one.

In contrast, in the context of digital rigid transformations (see Equation (4)), the way to restrict transformations under similar constraints is more permissive. Indeed, when setting the correspondence between one or several pairs of points $\left(\vec{p}_{i}, \vec{p}_{i}^{\prime}\right)$ of $\mathbb{Z}^{2}$, a larger space of rigid transformations remains valid (see 


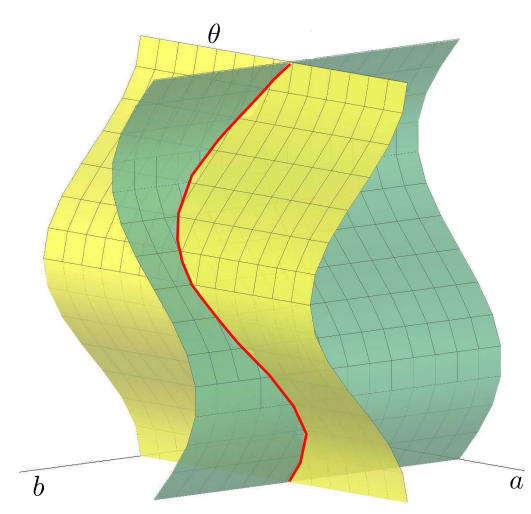

(a)

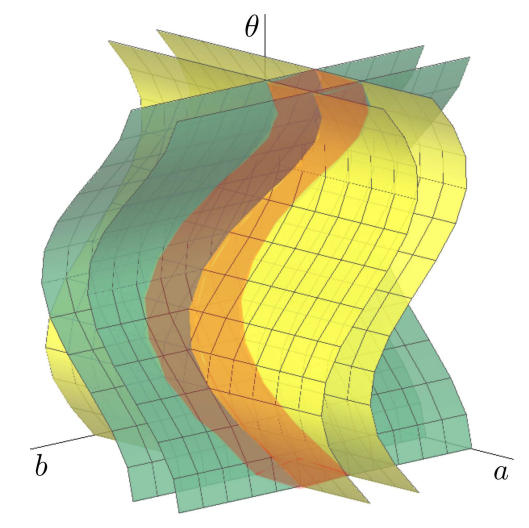

(c)

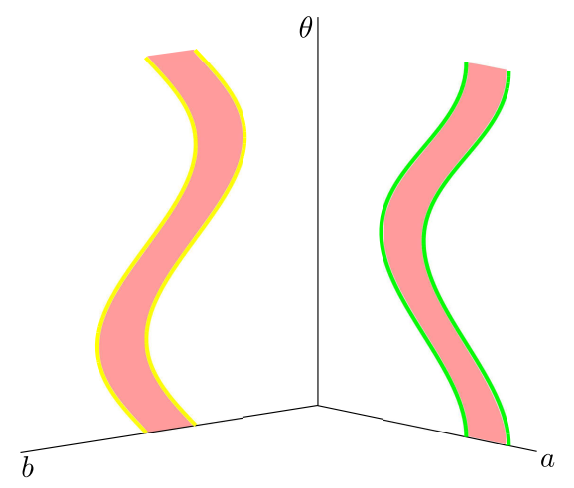

(e)

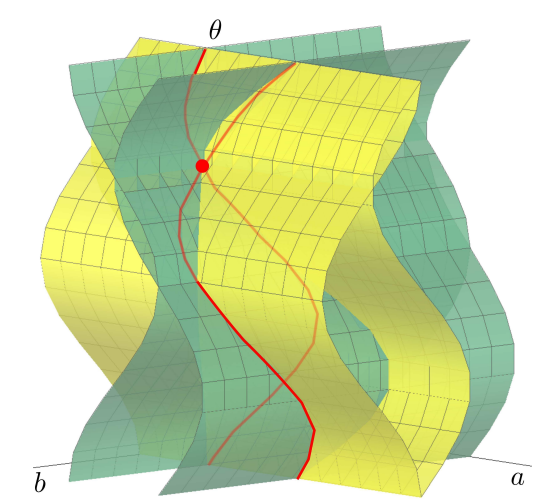

(b)

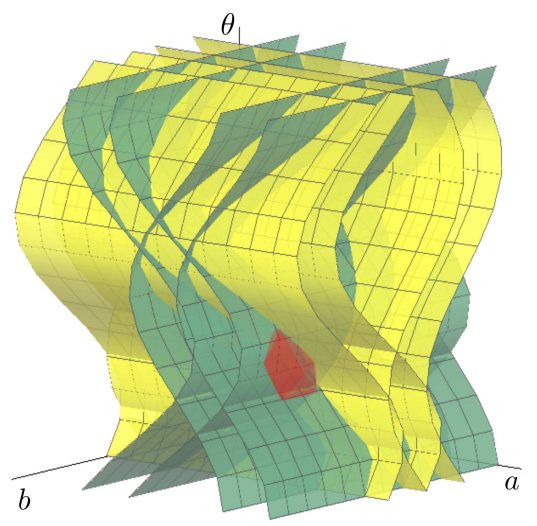

(d)

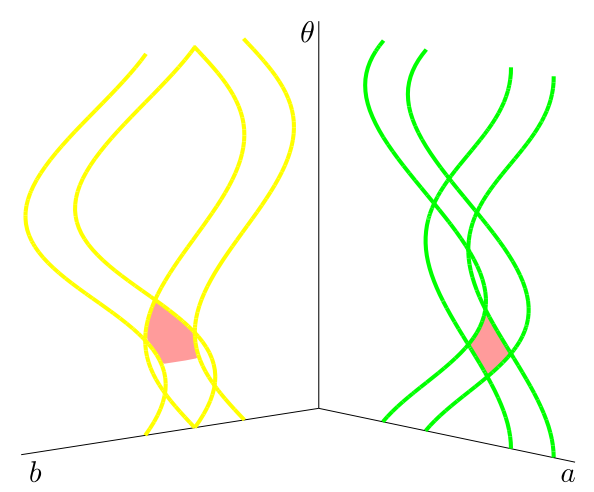

(f)

Figure 5: Feasible rigid transformations induced by geometric constraints in continuous $(a, b)$ and discrete $(c-f)$ frameworks. (a) Transformations with one point correspondence (red curve). (b) Transformation with two point correspondences (red dot at the intersection of the two red curves). (c) Transformations with one pixel correspondence (red tube-like volume). (d) Transformations with two pixel correspondences (red volume). (e,f) Projection/intersection of red volume parts of $(\mathrm{c}, \mathrm{d})$ respectively to the planes $(a, \theta)$ and $(b, \theta)$ with the associated tipping curves. 
Fig. 5(c,d)). Such constraints, which rely on the pixel decomposition of the image, are called pixel-invariance constraints and are defined as follows.

Definition 4 (Pixel invariance constraints) Let $\vec{p}=(p, q)$ and $\vec{p}=\left(p^{\prime}, q^{\prime}\right)$ in $\mathbb{S} \subset \mathbb{Z}^{2}$, where $\mathbb{S}$ is of size $N \times N$. There exists a pixel-invariance constraint between $\vec{p}$ and $\vec{p}$ if the authorised digital rigid transformations $T$ between $\vec{p}$ and $\overrightarrow{p^{\prime}}$ satisfy the equality $T(\vec{p})=\vec{p}$, i.e., if

$$
\begin{aligned}
& p^{\prime}-\frac{1}{2}<p \cos \theta-q \sin \theta+a<p^{\prime}+\frac{1}{2} \\
& q^{\prime}-\frac{1}{2}<p \sin \theta+q \cos \theta+b<q^{\prime}+\frac{1}{2}
\end{aligned}
$$

More generally, there exist pixel-invariance constraints between two sets $\left\{\vec{p}_{i}\right\}_{i=1}^{m}$ and $\left\{\vec{p}_{i}\right\}_{i=1}^{m}(m \geq 1)$ if $T\left(\vec{p}_{i}\right)=\vec{p}_{i}^{\prime}$ (i.e., if Inequalities (8)-(9) are satisfied) for every $i \in \llbracket 1, m \rrbracket$.

In the absence of constraints, the $3 \mathrm{D}$ parameter space $(a, b, \theta)$ induced by the subset of size $N \times N$ where the image is defined, is divided into cells whose boundaries are the tipping surfaces $\Phi_{p q k}$ and $\Psi_{p q l}$, with $p, q \in \llbracket 0, N-1 \rrbracket$ and $k, l \in \llbracket 0, N \rrbracket$. In this case, the whole parameter space models adequate rigid transformations.

Under a pixel-invariance constraint, some of the digital rigid transformations may become unfeasible. In other words, only a part of the parameter space namely the subspace of the parameters $(a, b, \theta)$ that satisfy this constraintremains valid. From the definition of pixel-invariance constraint, provided by Inequalities (8)-(9), this parameter subspace is defined by the intersection of four half-spaces associated to four tipping surfaces. This is visually illustrated in Fig. 5(c,e). The graph modelling the subdivision of such subspace is in particular a part of the whole DRT graph, induced by the pixel-invariance constraint.

\subsection{Feasible rigid transformation sets}

More generally, if a set $\mathcal{P}$ of $m$ pixel-invariance constraints is provided, the parameter subspace of relevant transformations is defined as the intersection of $m$ 3D regions induced by these constraints, i.e., as the intersection of $4 m$ half-spaces defined by Inequalities (8)-(9).

Let $p, q \in \llbracket 0, N-1 \rrbracket$ and $k, l \in \llbracket 0, N \rrbracket$. Let us consider the functions $V_{p q k}, H_{p q l}: \mathbb{R} \times[0,2 \pi[\rightarrow \mathbb{R}$ respectively defined by

$$
\begin{aligned}
& V_{p q k}(a, \theta)=a-\Phi_{p q k}(\theta) \\
& H_{p q l}(b, \theta)=b-\Psi_{p q l}(\theta)
\end{aligned}
$$

where $\Phi_{p q k}$ and $\Psi_{p q l}$ are two tipping surfaces (see Equations (5)-(6)). We then define the half-spaces induced by these tipping surfaces $\Phi_{p q k}$ and $\Psi_{p q l}$ with respect to inequalities (8)-(9) as

$$
\begin{aligned}
& V_{p q k}^{+}=\left\{(a, b, \theta) \mid V_{p q k}(a, \theta)>0\right\} \\
& V_{p q k}^{-}=\left\{(a, b, \theta) \mid V_{p q k}(a, \theta)<0\right\}
\end{aligned}
$$


and

$$
\begin{aligned}
& H_{p q l}^{+}=\left\{(a, b, \theta) \mid H_{p q l}(b, \theta)>0\right\} \\
& H_{p q l}^{-}=\left\{(a, b, \theta) \mid H_{p q l}(b, \theta)<0\right\}
\end{aligned}
$$

The notion of a feasible rigid transformation set is then defined as follows.

Definition 5 (Feasible rigid transformation set) Let $\mathcal{P}=\left\{\left(\vec{p}_{i}, \vec{p}_{i}^{\prime}\right)\right\}_{i=1}^{m}(m \geq$ 1) be a set of pixel-invariance constraints with $\vec{p}_{i}=\left(p_{i}, q_{i}\right)$ and $\vec{p}_{i}^{\prime}=\left(p_{i}^{\prime}, q_{i}^{\prime}\right)$ in $\mathbb{S} \subset \mathbb{Z}^{2}$. The feasible rigid transformation set (FRTS) associated to $\mathcal{P}$ is the subspace $\mathcal{R} \subset \mathbb{R}^{2} \times[0,2 \pi[$ of the parameter space $(a, b, \theta)$ defined by

$$
\mathcal{R}=\bigcap_{i \in \llbracket 1, m \rrbracket}\left(H_{p_{i} q_{i} p_{i}^{\prime}}^{+} \cap H_{p_{i} q_{i} p_{i}^{\prime}+1}^{-} \cap V_{p_{i} q_{i} q_{i}^{\prime}}^{+} \cap V_{p_{i} q_{i} q_{i}^{\prime}+1}^{-}\right)
$$

Note that for a single pixel-invariance constraint (i.e., for $m=1$ ), the FRTS forms a "tube" in the parameter space $(a, b, \theta)$ (see Fig. 5(c)). For two pixelinvariance constraints (i.e., for $m=2$ ), the FRTS forms a bounded and connected set (see Fig. 5(d)), or possibly becomes empty.

The FRTS is generated by $m$ pixel-invariance constraints, and divided into 3D cells whose boundaries are the tipping surfaces induced by the $\left(N^{2}-m\right)$ unconstrained pixels of the given image of size $N \times N$. It has to be recalled that each cell contains a set of rigid transformations that provide the same digital transformation, namely a discrete rigid transformation (DRT). In particular, the combinatorial structure modeling the subdivision of an FRTS into DRTs is represented by a part of the DRT graph, as defined in Section 2.3, and is called a feasible discrete rigid transformation graph (FDRT graph, for short). More explanation as well as the construction of this graph is given in Section 4.

We now introduce the notion of directional convexity, and show that any FRTS is directionally convex. This property will be used in the next section to study the combinatorial structure of DRTs under pixel-invariance constraints.

Definition 6 (Directional convexity) $A$ region $R \subseteq \mathbb{R}^{n}$ in an $n$-variable space $\left(x_{1}, \ldots, x_{n}\right)$ is $x_{k}$-convex if, for any two points $\overrightarrow{p_{1}}, \overrightarrow{p_{2}} \in R$ such that the segment $\left[\overrightarrow{p_{1}} \overrightarrow{p_{2}}\right]=\left\{\alpha \cdot \overrightarrow{p_{1}}+(1-\alpha) \cdot \overrightarrow{p_{2}} \mid \alpha \in[0,1]\right\}$ is parallel to the $x_{k}$-axis, $\left.\overrightarrow{p_{1}} \overrightarrow{p_{2}}\right]$ is included in $R$.

Property 7 Any FRTS is both $a$ - and b-convex in the space $(a, b, \theta)$.

Proof This is a direct consequence of the fact that any FRTS is the intersection of half-spaces which are both $a$ - and $b$-convex (see Equation (16)).

Based on the relations that link tipping surfaces and tipping curves (see Equations (5)-(6) and Figs. 2 and 5(c-f)), it is plain that an FRTS, defined in the parameter space $(a, b, \theta)$ by Equation (16), can be fully described from its 
two projections $\mathcal{R}_{H}$ and $\mathcal{R}_{V}$ on the planes $(a, \theta)$ and $(b, \theta)$ respectively, defined as

$$
\begin{aligned}
& \mathcal{R}_{H}=\bigcap_{i \in \llbracket 1, m \rrbracket}\left(h_{p_{i} q_{i} q_{i}^{\prime}}^{+} \cap h_{p_{i} q_{i} q_{i}^{\prime}+1}^{-}\right) \\
& \mathcal{R}_{V}=\bigcap_{i \in \llbracket 1, m \rrbracket}\left(v_{p_{i} q_{i} p_{i}^{\prime}}^{+} \cap v_{p_{i} q_{i} p_{i}^{\prime}+1}^{-}\right)
\end{aligned}
$$

where $h_{p q l}^{*}$ (resp. $v_{p q k}^{*}$ ) is the cross-section of $H_{p q l}^{*}$ (resp. $V_{p q k}^{*}$ ) with the plane $(a, \theta)$ (resp. $(b, \theta))$. In this context, $h_{*}^{+}, v_{*}^{+}$are called upper half-planes and $h_{*}^{-}, v_{*}^{-}$lower half-planes.

From Property 7 , it is obvious that $\mathcal{R}_{\mathcal{H}}$ (resp. $\mathcal{R}_{\mathcal{V}}$ ) has a pair of upper and lower half-planes $\left(h^{+}, h^{-}\right)$(resp. $\left.\left(v^{+}, v^{-}\right)\right)$as the upper and lower parts of the boundary for each $\theta$. Thus, the boundary of $\mathcal{R}_{\mathcal{H}}$ (resp. $\mathcal{R}_{\mathcal{V}}$ ) consists of two sets of half-planes:

- the upper boundary $\mathbf{U}=\left\{h_{p_{i} q_{i} q_{i}^{\prime}}, \ldots\right\}$ containing only upper half-planes;

- the lower boundary $\mathbf{L}=\left\{h_{p_{i} q_{i} q_{i}^{\prime}+1}^{-}, \ldots\right\}$ containing only lower half-planes.

From Property 7, we can also derive the following corollary for $\mathcal{R}_{\mathcal{H}}$; a similar corollary is established for $\mathcal{R}_{\mathcal{V}}$ as well.

Corollary 8 Let $\mathcal{R}_{\mathcal{H}}$ be the projection of an FRTS $\mathcal{R}$, and $\mathbf{U}$ (resp. $\mathbf{L}$ ) be the upper (resp. lower) boundary of $\mathcal{R}_{\mathcal{H}}$. Then $\mathbf{U}$ (resp. $\left.\mathbf{L}\right)$ always contains at least one upper (resp. lower) half-plane.

We now derive the following result related to the connectedness of an FRTS, which will be useful in the following section. Here, instead of the parameter space $\mathbb{R}^{2} \times[0,2 \pi[$ of $(a, b, \theta)$, we consider -without loss of correctness- the quotient space $\mathbb{R}^{3} / \sim$ where $(a, b, \theta) \sim(a, b, \theta+2 \pi)$.

Property 9 An FRTS is connected in the quotient space $\mathbb{R}^{3} / \sim$ of the parameter space $(a, b, \theta)$.

Proof If $m=1$, it derives from Inequalities (8)-(9) that the FRTS is the Minkowski addition between a 1D (connected) trigonometric curve-defined as a function from the $(a, b)$ space to the $\theta$ one- and a (connected) square pattern ]$-\frac{1}{2}, \frac{1}{2}\left[{ }^{2} \subset \mathbb{R}^{2}\right.$ defined in the $(a, b)$ space. It straightforwardly derives that the FRTS is then connected.

Let us now suppose that $m=2$. Let $\left(\vec{p}_{i}, \vec{p}_{i}^{\prime}\right)$, for $i=1,2$, be the two pixelinvariance constraints that generate the FRTS $\mathcal{R}$. As explained above, $\mathcal{R}$ can be described from its two projections $\mathcal{R}_{H}$ and $\mathcal{R}_{V}$ on the planes $(a, \theta)$ and $(b, \theta)$ respectively by tipping curves. Let us first consider $\mathcal{R}_{H}$; from Equation (17), $\mathcal{R}_{H}$ has two upper half-planes $\mathbf{U}=\left\{h_{p_{1} q_{1} q_{1}^{\prime}}^{+}, h_{p_{2} q_{2} q_{2}^{\prime}}^{+}\right\}$and two lower half-planes $\mathbf{L}=\left\{h_{p_{1} q_{1} q_{1}^{\prime}+1}^{-}, h_{p_{2} q_{2} q_{2}^{\prime}+1}^{-}\right\}$. Then it is easily seen that any pair of upper and lower half-planes of $\mathcal{R}_{H},\left(h^{+}, h^{-}\right) \in \mathbf{U} \times \mathbf{L}$, has the intersection $h^{+} \cap h^{-}$that is 
connected in $\mathbb{R}^{2} / \sim$ of the parameter space $(a, \theta)$, where $(a, \theta) \sim(a, \theta+2 \pi)$, as the associated tipping curves have at most two intersections for $\theta \in[0,2 \pi[$ (see $[17$, Prop. 2, Cor. 2]). For instance, each of $h_{p_{1} q_{1} q_{1}^{\prime}}^{+} \cap h_{p_{2} q_{2} q_{2}^{\prime}+1}^{-}$and $h_{p_{2} q_{2} q_{2}^{\prime}}^{+} \cap h_{p_{1} q_{1} q_{1}^{\prime}+1}^{-}$ is a connected region in the quotient space $\mathbb{R}^{2} / \sim$. However, the intersection of those two regions, i.e., $\mathcal{R}_{H}$ may give at most two connected regions in the quotient space $\mathbb{R}^{2} / \sim$. A similar result is obtained for $\mathcal{R}_{V}$. We now show that the $3 \mathrm{D}$ intersection of (the extrusion of) $\mathcal{R}_{H}$ and $\mathcal{R}_{V}$ gives only one admissible connected region in the quotient space $\mathbb{R}^{3} / \sim$. As we know that the intersection of $\mathcal{R}_{H}$ and $\mathcal{R}_{V}$, i.e. $\mathcal{R}$, is never empty, there always exist $\left.\left(r_{i}, s_{i}\right) \in\right] p_{i}^{\prime}-\frac{1}{2}, p_{i}^{\prime}+$ $\frac{1}{2}[\times] q_{i}^{\prime}-\frac{1}{2}, q_{i}^{\prime}+\frac{1}{2}[$ for $i=1,2$ such that

$$
\begin{aligned}
& r_{1}=p_{1} \cos \theta-q_{1} \sin \theta+a \\
& s_{1}=p_{1} \sin \theta-q_{1} \cos \theta+b \\
& r_{2}=p_{2} \cos \theta-q_{2} \sin \theta+a \\
& s_{2}=p_{2} \sin \theta-q_{2} \cos \theta+b
\end{aligned}
$$

At any intersection between (19) and (21) in the plane $(a, \theta)$, the following equation must be satisfied:

$$
K-P \cos \theta+Q \sin \theta=0
$$

by setting $P=p_{1}-p_{2}, Q=q_{1}-q_{2}$ and $K=r_{1}-r_{2}$. Similarly, at any intersection between $(20)$ and $(22)$ in the plane $(b, \theta)$, the following equation must be satisfied:

$$
L-P \sin \theta-Q \cos \theta=0
$$

by additionally setting $L=s_{1}-s_{2}$. The system of linear equations $(23),(24)$ has a determinant equal to $P^{2}+Q^{2} \neq 0$, since the two pixel-invariance constraints are distinct. It then admits exactly one solution for the pair of unknowns $\cos \theta$ and $\sin \theta$, and thus at most one solution for $\theta$ in $[0,2 \pi[$. Since the FRTS $\mathcal{R}$ contains such a solution, $\mathcal{R}$ must be connected. Finally, the result for $m>2$ follows by induction.

\section{Combinatorial structure of feasible discrete rigid transformations}

An FRTS contains the rigid transformations that satisfy some given pixelinvariance constraints. It can then be subdivided into DRTs (see Section 2.3). This section presents a method for constructing the combinatorial structure of DRTs in an FRTS (namely, the FDRT graph) by following three successive steps: (i) finding the boundaries of the FRTS in the parameter space (Section 4.2); (ii) finding the tipping surfaces passing through this FRTS (Section 4.3) and their intersecting points (Section 4.4); (iii) constructing the associated DRT graph (Section 4.5). Before describing these steps, we first recall an algorithm (Section 4.1) useful for building a graph modeling a subdivision of the parameter space from a given set of tipping surfaces without considering the FRTS. 


\subsection{Incremental construction of a discrete rigid transfor- mation graph based on a sweeping method}

In [17], we showed how to build a DRT graph using an incremental algorithm, that mainly relies on the algorithmic notion of surface arrangement [5, 25]. A surface arrangement is defined as a decomposition of the space $\mathbb{R}^{3}$ into cells by a finite set of surfaces. Such a decomposition generates four types of cells: 0-D vertices, 1-D arcs, 2-D surfaces and 3-D regions.

This algorithm can be extended to deal with supplementary constraints such as those discussed in the previous section. The resulting graph $G$, called FDRT graph, actually extends the notion of DRT graph initially introduced in [17]. Given a set of tipping surfaces $S$, we then need to construct the graph modeling the subdivision of the parameter space $(a, b, \theta)$ induced by $S$. In $G$, each vertex remains associated to a $3 \mathrm{D}$ open cell of the subdivision, and each tipping-surface segment shared by two adjacent 3D open cells, is associated to an edge.

Surface arrangement algorithms present a polynomial complexity $\Omega\left(n^{4}\right)$ [25], where $n$ is the number of surfaces. However, we are only interested in the information of regions ( $3-\mathrm{D}$ cells) and faces (2-D cells) in the arrangement. In this specific case -when the surfaces are tipping surfaces- a better complexity for building the DRT graph in $\mathcal{O}\left(n^{3}\right)$ can be achieved [17].

As described in Section 2.2, while projecting two families of tipping surfaces on the planes $(a, \theta)$ and $(b, \theta)$, we obtain the corresponding families of tipping curves defined by Formulae (5)-(6) (see Fig. 2). Relying on this property, the subdivision of the parameter space $(a, b, \theta)$ by these surfaces can be fully described from their two cross-sections in the planes $(a, \theta)$ and $(b, \theta)$, respectively expressed by two sets of tipping curves [17]. This leads to a constructive algorithm with a better complexity. More precisely, we first consider the structure of the graphs in the 2D planes $(a, \theta)$ and $(b, \theta)$ planes, and then combine them to build the complete DRT graph. In the sequel, we briefly recall the principles of this algorithm and its implementation (degenerate cases will not be discussed here; these details can be found in [17].)

We first define a cut for a plane -either $(a, \theta)$ or $(b, \theta)$ - denoted by $\gamma$, as a monotonic line [7] intersecting exactly once each tipping curve in the plane. The monotonic property of the cut is a result of $a$ - and $b$-convexity in the space $(a, b, \theta)$ (see Property 7 ). A cut is then modeled by its sequence of intersecting tipping curves (see Fig. 6). This sequence can be conveniently represented as a directed graph as follows.

Definition 10 Let $\gamma=\left(\phi_{1}, \phi_{2}, \ldots\right)$ be a sequence corresponding to a cut, where $\phi_{1}, \phi_{2}, \ldots$ are tipping curves. A graph $G_{\gamma}=\left(V_{\gamma}, E_{\gamma}\right)$ with respect to $\gamma$ consists of

- a set of vertices $V_{\gamma}=\left\{v_{0}, v_{1}, \ldots\right\}$; and

- a set of labelled edges $E_{\gamma}=\left\{\left(v_{0}, v_{1}, \phi_{1}\right),\left(v_{1}, v_{2}, \phi_{2}\right), \ldots\right\}$, for which each edge $(u, w, f) \in E_{\gamma}$ connects two vertices $u, w \in V_{\gamma}$ separated by the tipping curve $f$, which is considered as an edge label. 

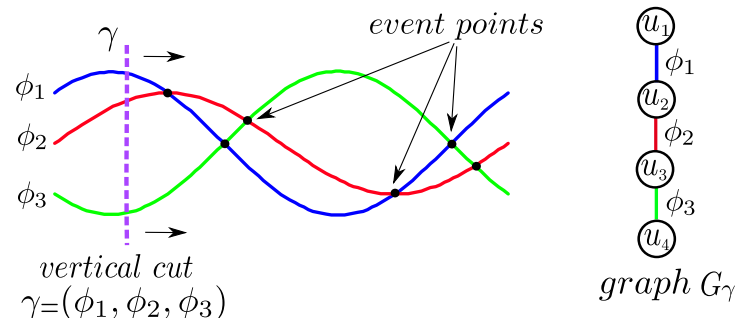

Figure 6: Example of a cut $\gamma$ and its associated graph $G_{\gamma}$.
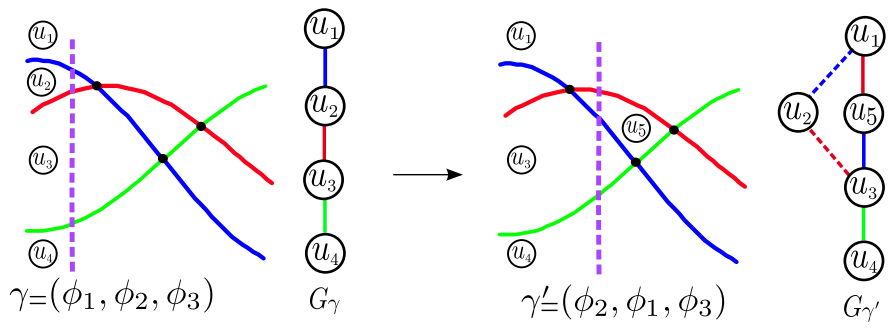

Figure 7: Progress of the cut at an event point by which the cut is updated and the corresponding graph is modified.

Practically, the elements of $E_{\gamma}$ are ordered in the same way as $\gamma$ (see Fig. 6).

The sweeping method in 2D then consists of sweeping a cut $\gamma$ across all tipping curves in the plane from $\theta=0$ to $2 \pi$. While moving the cut, its sequence changes only at intersections of tipping curves, called event points. When a cut reaches an event point, the algorithm performs an update of its sequence, and generates new vertices and edges in the graph (see Fig. 7). This constitutes an elementary step of the algorithm. Practically, it is only required to maintain a set of sorted event points with respect to $\theta$, and to progress the cut in their increasing order to build the graph incrementally.

For building a DRT graph $G$ in the 3D parameter space $(a, b, \theta)$, two cuts are used such that each cut sweeps in either the plane $(a, \theta)$ or $(b, \theta)$. We denote those cuts by $\gamma_{a}$ and $\gamma_{b}$ respectively. For each update of the cuts, $\gamma_{a}$ and $\gamma_{b}$, the associated graphs, $G_{\gamma_{a}}$ and $G_{\gamma_{b}}$, are respectively modified, so that a part of $G$ is generated. We call such a part of $G$ a partial graph, denoted by $\delta G$. In fact, $\delta G$ is a combination of the two graphs $G_{\gamma_{a}}$ and $G_{\gamma_{b}}$ (see Fig. 8).

Definition 11 The partial graph $\delta G=(\delta V, \delta E)$ is generated from $G_{\gamma_{a}}=$ $\left(V_{\gamma_{a}}, E_{\gamma_{a}}\right)$ and $G_{\gamma_{b}}=\left(V_{\gamma_{b}}, E_{\gamma_{b}}\right)$, such that

- $\delta V=\left\{\left(v_{a}, v_{b}\right) \mid v_{a} \in V_{\gamma_{a}}, v_{b} \in V_{\gamma_{b}}\right\}$, and

- $\delta E=\left\{\left(\left(u_{1}, v\right),\left(u_{2}, v\right), \phi_{u}\right) \mid u_{1}, u_{2} \in V_{\gamma_{a}}, v \in V_{\gamma_{b}},\left(u_{1}, u_{2}, \phi_{u}\right) \in E_{\gamma_{a}}\right\} \cup$ $\left\{\left(\left(u, v_{1}\right),\left(u, v_{2}\right), \phi_{v}\right) \mid v_{1}, v_{2} \in V_{\gamma_{b}}, u \in V_{\gamma_{a}},\left(v_{1}, v_{2}, \phi_{v}\right) \in E_{\gamma_{b}}\right\}$. 


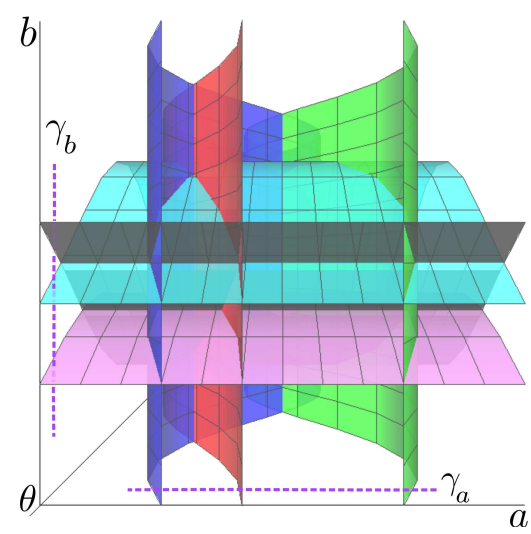

(a)

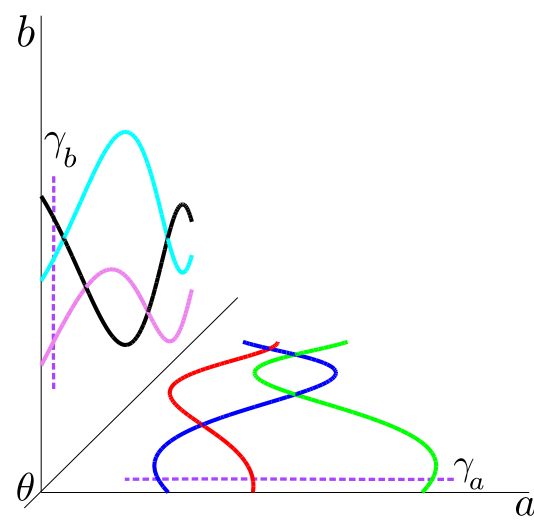

(b)

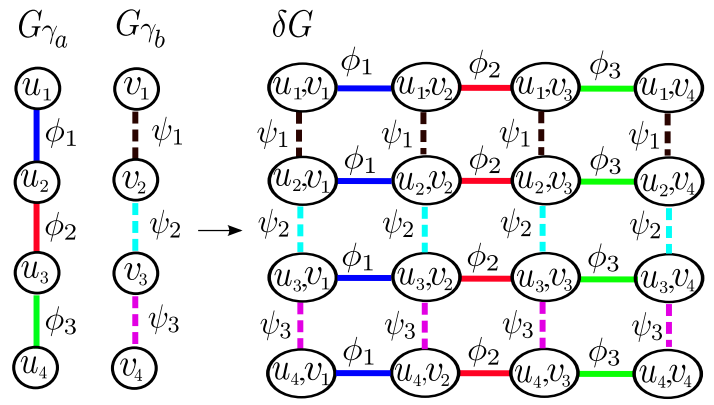

(c)

Figure 8: Generation of a partial graph $\delta G$ from two graphs $G_{\gamma_{a}}$ and $G_{\gamma_{b}}$ associated to cuts $\gamma_{a}$ and $\gamma_{b}$ respectively (see Definition 11). 
When the $i$-th elementary step is applied to $G_{\gamma_{a}}$ or $G_{\gamma_{b}}$, the sweep progresses as the partial graph $\delta G_{i}$ is generated and integrated in $G$ for constructing the final graph as well. The following proposition was originally proposed in [17].

Proposition 12 Let $S$ be a set of tipping surfaces, e be the total number of ordered event points, and $G$ be a DRT graph modeling the subdivision of the parameter space by $S$. We have

$$
G=\bigcup_{i \in \llbracket 1, e \rrbracket} \delta G_{i}
$$

where $\delta G_{i}$ is a partial graph at the $i$-th elementary step.

Note that a partial graph $\delta G_{i}$ is a directed graph because we require the edge direction information during its generation. However the final graph $G$ is not directed, so that we do not keep directions while integrating $\delta G_{i}$ into $G$. More details about the sweeping algorithm for tipping surfaces can be found in [17].

In the partial graph $\delta G_{i}$, if there exists a directed edge from a vertex $u$ to another vertex $v$, then $u$ is called a predecessor of $v$ and $v$ is a successor of $u$. Let us call a vertex a top (resp. bottom) vertex if it has no predecessors (resp. no successors), and a middle vertex, otherwise. The following property straightforwardly derives from the DRT graph construction algorithm.

Property 13 Every partial graph generated during the construction of a DRT graph $G$ has only one top (resp. bottom) vertex, and this vertex does not change during the construction of $G$. This remains true for the directed graphs of the cuts $G_{\gamma_{a}}$ and $G_{\gamma_{b}}$.

\subsection{Finding the boundary of a feasible rigid transforma- tion set}

It is possible to describe an FRTS $\mathcal{R}$, defined from a set of $m$ pixel-invariance constraints $\mathcal{P}=\left\{\left(\vec{p}_{i}, \vec{p}_{i}^{\prime}\right)\right\}_{i=1}^{m}$, using a set of half-spaces constituting only the boundary of $\mathcal{R}$, instead of using all the half-spaces from $\mathcal{P}$ in Definition 5 . This section explains how to find such a set of half-spaces, by using the above sweeping algorithm.

As explained in Section 3.2, an FRTS $\mathcal{R}$ induced by $\mathcal{P}$ in the parameter space $(a, b, \theta)$ can be fully described from its two cross-sections $\mathcal{R}_{H}$ and $\mathcal{R}_{V}$ on the planes $(a, \theta)$ and $(b, \theta)$ as defined in Equations (17)-(18), and illustrated in Fig. 5.

Relying on the similarity of $\mathcal{R}_{\mathcal{H}}$ and $\mathcal{R}_{\mathcal{V}}$, hereafter we consider only $\mathcal{R}_{\mathcal{H}}$ (the same argument stands for $\mathcal{R}_{\mathcal{V}}$ ). Our problem is then specified as follows: given a constraint set $\mathcal{P}$ of half-planes of $\mathcal{R}_{\mathcal{H}}$, report the set of half-planes constituting the boundary of $\mathcal{R}_{\mathcal{H}}$. From Corollary 8 and Property 9 , we recall that $\mathcal{R}_{\mathcal{H}}$ contains two non-empty sequences of half-planes:

- a upper-boundary sequence $U=\left(h_{p_{i} q_{i} q_{i}^{\prime}}^{+}, \ldots\right)$ that contains only the upper half-planes; and 


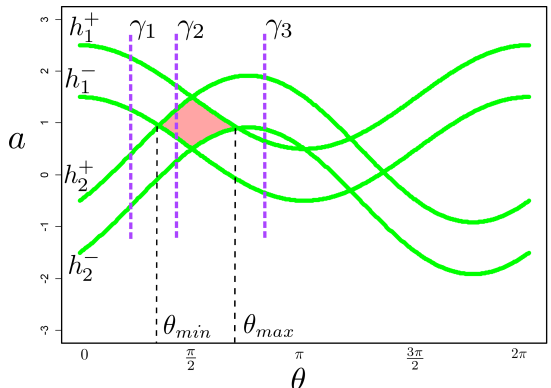

(a)

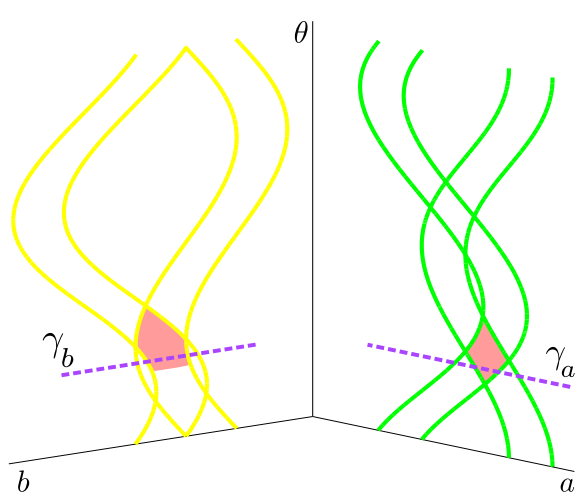

(b)

Figure 9: (a) Progression of a cut $\gamma$ in the cross-section $\mathcal{R}_{\mathcal{H}}$ of an FRTS $\mathcal{R}$ (in red) on the plane $(a, \theta)$. The initial cut is $\gamma_{1}=\left(h_{1}^{+}, h_{1}^{-}, h_{2}^{+}, h_{2}^{-}\right)$. When it crosses $\mathcal{R}_{\mathcal{H}}$, it becomes $\gamma_{2}=\left(h_{1}^{+}, h_{2}^{+}, h_{1}^{-}, h_{2}^{-}\right)$. It then becomes $\gamma_{3}=\left(h_{2}^{+}, h_{2}^{-}, h_{1}^{+}, h_{1}^{-}\right)$ when leaving $\mathcal{R}_{\mathcal{H}}$. (b) Cross-sections of the constraints on the planes $(a, \theta)$ and $(b, \theta)$ via the use of tipping curves.

- a lower-boundary sequence $L=\left(h_{p_{i} q_{i} q_{i}^{\prime}+1}^{-}, \ldots\right)$ that contains only the lower half-planes.

The 2D sweeping algorithm ${ }^{3}$, presented in Section 4.1, is used to find the $U$ and $L$ of $\mathcal{R}_{\mathcal{H}}$, such that the cut $\gamma$ is now represented as a sequence of halfplanes intersecting it. Note that no FDRT graph is built at this stage; we only need to observe the sequence of the cut $\gamma$ during its update in order to obtain all the elements of $U$ and $L$. Indeed, while sweeping $\gamma$, its sequence changes at event points. We remark that $\gamma$ intersects with $\mathcal{R}_{\mathcal{H}}$ when its sequence of half-planes is separated into two sequences of $\gamma^{+}$and $\gamma^{-}$, namely $\gamma=\gamma^{+} \gamma^{-}$, where $\gamma^{+}$contains only the upper half-planes and $\gamma^{-}$contains only the lower half-planes. Moreover, we see that the last element of $\gamma^{+}$and the first element of $\gamma^{-}$correspond to the upper and lower half-planes constituting the boundary of $\mathcal{R}_{\mathcal{H}}$ respectively. The cut is moved out of $\mathcal{R}_{\mathcal{H}}$ when there is no longer any such separation. Under the change of $\gamma$ in $\mathcal{R}_{\mathcal{H}}$, an upper or lower half-plane is progressively added in $U$ and $L$ at each event point, as illustrated in Fig. 9(a).

By using two cuts $\gamma_{a}$ and $\gamma_{b}$ sweeping in the two planes $(a, \theta)$ and $(b, \theta)$, we can find the boundary of an FRTS $\mathcal{R}$. Indeed, at each event point either on $(a, \theta)$ or $(b, \theta)$, the algorithm updates and checks the sequences of both cuts. We start getting the boundary segments of $\mathcal{R}$ from the first $\theta$ at which both sequences of $\gamma_{a}$ and $\gamma_{b}$ are separated in two parts. Similarity, we stop collecting the boundary segments of $\mathcal{R}$ at the first $\theta$ at which there is no longer any such separation in $\gamma_{a}$ or $\gamma_{b}$, as illustrated in Fig. 9(b). Moreover, from this procedure we can also obtain the lowest and greatest values of $\theta$ of $\mathcal{R}$, denoted respectively

\footnotetext{
${ }^{3}$ The algorithm can easily modified to deal with the quotient space $\mathbb{R}^{3} / \sim$.
} 


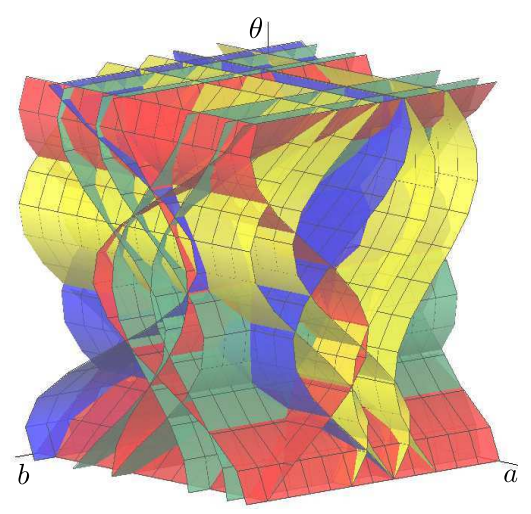

(a)

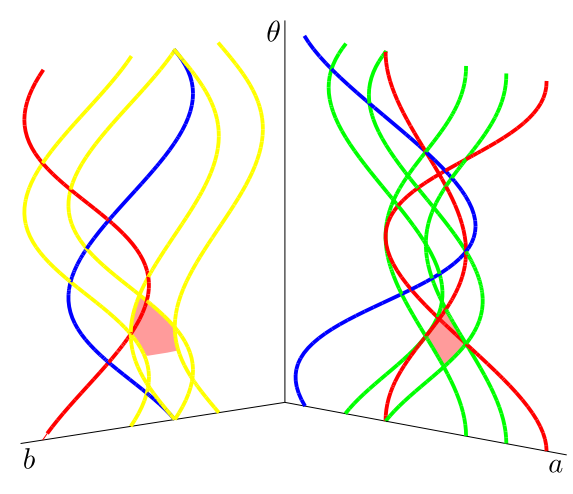

(b)

Figure 10: (a) Example of tipping surfaces passing through $\mathcal{R}$ (in red) and not passing through $\mathcal{R}$ (in blue) in the parameter space $(a, b, \theta)$. (b) Its crosssections $\mathcal{R}_{\mathcal{H}}$ and $\mathcal{R}_{\mathcal{V}}$ of (a) on the planes $(a, \theta)$ and $(b, \theta)$ respectively.

by $\theta_{\min }$ and $\theta_{\max }$, which are needed in the next stage of the algorithm. From Properties 7 and 9 , we know that these values $\theta_{\min }$ and $\theta_{\max }$ of $\mathcal{R}$ are unique.

As stated above, we use this sweeping method in order to compute an FDRT graph modeling the subdivision of an FRTS $\mathcal{R}$ into DRTs. For this, we require not only the tipping surfaces constituting the boundary of $\mathcal{R}$ but also those passing through $\mathcal{R}$. We explain how to find such tipping surfaces in the next section.

\subsection{Finding tipping surfaces passing through a feasible rigid transformation set}

So far, we know that an FRTS $\mathcal{R}$ contains all the rigid transformations satisfying given pixel-invariance constraints. $\mathcal{R}$ is partitioned into DRTs as well as the whole parameter space of rigid transformations, as explained in Section 2.3; the subdivision of $\mathcal{R}$ is induced by the tipping surfaces existing in $\mathcal{R}$ (see Fig. 10(a)). Therefore, we need to determine such surfaces among all vertical and horizontal tipping surfaces $\Phi_{p q k}$ and $\Psi_{p q l}$ respectively, for $p, q \in \llbracket 0, N-1 \rrbracket$ and $k, l \in \llbracket 0, N \rrbracket$, where $N \times N$ is the image size. This problem is equivalent to finding the tipping curves $\phi_{p q k}$ (resp. $\psi_{p q l}$ ) passing through $\mathcal{R}_{\mathcal{H}}$ (resp. $\mathcal{R}_{\mathcal{V}}$ ), the cross-sections of $\mathcal{R}$ on the plane $(a, \theta)$ (resp. $(b, \theta)$ ) (see Fig. $10(\mathrm{~b})$ ).

Let us consider the cross-section $\mathcal{R}_{\mathcal{H}}$ of $\mathcal{R}$. We call a segment of tipping curves that constitutes the boundary of $\mathcal{R}_{\mathcal{H}}$ a boundary segment. Any tipping curve $\phi_{p q k}$ passes $\mathcal{R}_{\mathcal{H}}$ if it intersects one of the boundary segments of $\mathcal{R}_{\mathcal{H}}$, without loss of generality, we denote such a boundary segment $\phi_{p^{\prime} q^{\prime} k^{\prime}}$. This is easily detected by verifying the relationship between $\phi_{p q k}$ and $\phi_{p^{\prime} q^{\prime} k^{\prime}}$, and the intersection is on a boundary segment of $\mathcal{R}_{\mathcal{H}}$ as follows: 
(i) verify if $\phi_{p q k}$ and $\phi_{p^{\prime} q^{\prime} k^{\prime}}$ intersect; this is true iff the following relations are satisfied [17, Property 2]:

$$
\begin{aligned}
\Delta_{1}+\Delta_{2} & >0 \\
\left|K P \pm \sqrt{\Delta_{1}}\right| & \leq P^{2}+Q^{2} \\
\left|K Q \pm \sqrt{\Delta_{2}}\right| & \leq P^{2}+Q^{2}
\end{aligned}
$$

where $P=p-p^{\prime}, Q=q-q^{\prime}, K=k-k^{\prime}, \Delta_{1}=P^{2}\left(P^{2}+Q^{2}-K^{2}\right)$ and $\Delta_{2}=Q^{2}\left(P^{2}+Q^{2}-K^{2}\right)$

(ii) if they intersect, then calculate the following values at the intersection $[17$, Corollary 1]:

$$
\begin{aligned}
& \sin \theta=\frac{K Q \pm \sqrt{\Delta_{1}}}{P^{2}+Q^{2}} \\
& \cos \theta=\frac{K P \pm \sqrt{\Delta_{2}}}{P^{2}+Q^{2}}
\end{aligned}
$$

and verify if $\theta_{\min } \leq \theta \leq \theta_{\max }$, where $\theta_{\min }$ and $\theta_{\max }$ are obtained from Section 4.2 ;

(iii) if (ii) is verified, then calculate

$$
\begin{aligned}
& a_{\text {upper }}=\max _{h_{p q k}^{+} \in U}\left\{\phi_{p q k}(\theta)\right\} \\
& a_{\text {lower }}=\min _{h_{p q k}^{-} \in L}\left\{\phi_{p q k}(\theta)\right\}
\end{aligned}
$$

and verify if $a_{\text {upper }} \leq a \leq a_{\text {lower }}$, where the value $a$ at the above intersection $\theta$ is calculated from Equation (5).

Note that the values $\cos \theta$ and $\sin \theta$ are used to represent $\theta$. Since all $\cos \theta$, $\sin \theta, \cos \theta_{\text {min }}, \sin \theta_{\text {min }}, \cos \theta_{\max }, \sin \theta_{\max }, a, a_{\min }$ and $a_{\max }$ are quadratic irrationals ${ }^{4}$, they can be compared exactly in (average) constant time [24].

\subsection{Determination of event points in a feasible rigid trans- formation set}

When carrying out the sweeping algorithm, it is mandatory to know how to detect event points in $\mathcal{R}$, or -equivalently- when to perform an elementary step. Due to the similarity of $\mathcal{R}_{\mathcal{V}}$ and $\mathcal{R}_{\mathcal{H}}$, in the following we consider only the cross-section $\mathcal{R}_{\mathcal{H}}$ of $\mathcal{R}$. Event points in $\mathcal{R}_{\mathcal{H}}$ are defined as intersections of tipping-curve segments constituting the boundary of $\mathcal{R}_{\mathcal{H}}$ (see Section 4.2) and those passing through $\mathcal{R}_{\mathcal{H}}$ (see Section 4.3), as illustrated in Fig. 11. We denote by $\mathcal{C}$ the set of tipping curves that bound or pass through $\mathcal{R}_{\mathcal{H}}$. Thus, event

\footnotetext{
${ }^{4} \mathrm{~A}$ quadratic irrational is an irrational number that is a solution of some quadratic equations.
} 


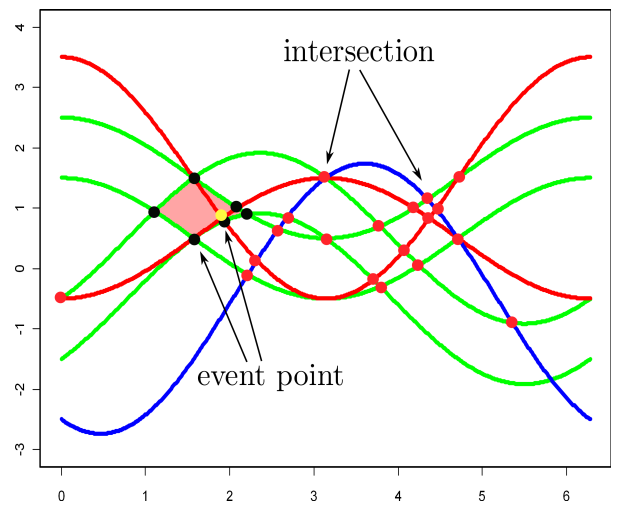

Figure 11: Illustration of boundary event points (in black), interior event points (in yellow) and intersections of tipping curves but not event points (in red).

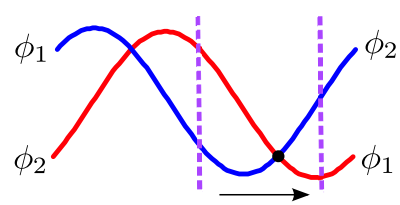

(a)

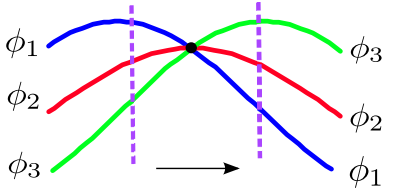

(b)

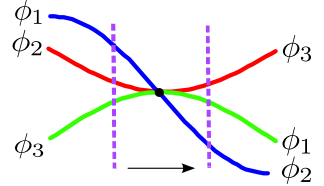

(c)

Figure 12: Classification of interior event points: a simple intersection if it is generated by only two tipping curves (a), otherwise it is a degeneracy (b,c), i.e., when there are more than two tipping curves.

points with respect to $\mathcal{R}_{\mathcal{H}}$ generated by $\mathcal{C}$ can be either on a boundary segment or interior of $\mathcal{R}_{\mathcal{H}}$, otherwise we simply have intersections (see Fig. 11). The procedure for handling event points in $\mathcal{R}_{\mathcal{H}}$ is explained in Section 4.5. We now focus on how to detect the event points.

According to the nature of an event point, it is called either a boundary event point or an interior event point. Similarly to the previous method in Section 4.3, if an intersection coordinate $(\theta, a)$ satisfies $\theta_{\min } \leq \theta \leq \theta_{\max }$ and $a_{\text {lower }}(\theta) \leq a \leq a_{\text {upper }}(\theta)$, then it is an event point in $\mathcal{R}_{\mathcal{H}}$. The algorithm described in Section 4.1 deals with any interior event points. In contrast, the boundary event points must be treated separately as follows.

In [17], we detected and classified interior event points into simple and degenerate cases (see Fig. 12). More precisely, we have

- simple cases: only two tipping curves intersect at an event point;

- degenerate cases: more than two tipping curves intersect (or, are intersecting and/or tangent) at an event point.

Regarding boundary event points, they can be classified into the following six types. We have, as illustrated in Fig. 13, an event point that: 


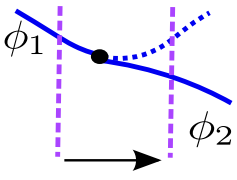

(a)

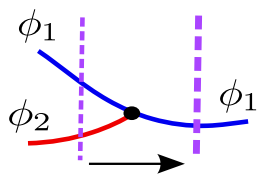

(d)

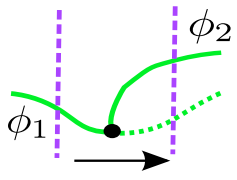

(b)

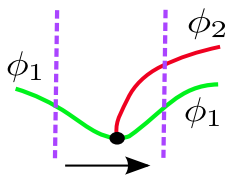

(e)

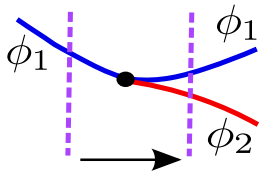

(c)

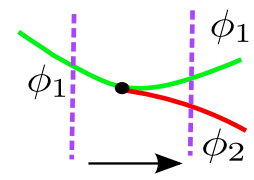

(f)

Figure 13: Classification of simple boundary event points, an event point with a tipping curve that: changes a boundary $(\mathrm{a}, \mathrm{b})$, goes in and out by an upper boundary $(\mathrm{c}, \mathrm{d})$, goes in and out by a lower boundary (e,f). Upper and lower boundaries are colored in blue and red respectively.

- changes the boundary segment, which is either upper (type a) or lower (type b);

- does not change the boundary segment, such that one of the tipping curves

- goes into (resp. leaves) $\mathcal{R}$ by the upper boundary segment (type c) (resp. (type d));

- goes into (resp. leaves) $\mathcal{R}$ by the lower boundary segment (type e) (resp. (type f)).

Following this classification, the type of a boundary event point can be easily detected as follows. Let $\mathbf{q}$ be a boundary event point, $\mathbf{q}$ is presented as the set of tipping curves intersecting at $\mathbf{q}$ [17], i.e., in simple case $\mathbf{q}=\left\{\phi_{u}, \phi_{v}\right\}$. Let $\gamma\left(\phi_{1}, \phi_{2}, \ldots, \phi_{n-1}, \phi_{n}\right)$ be the cut on the left of q. Let $U$ and $L$ be respectively the upper and lower bound sequences, and $\phi_{1} \in U$ and $\phi_{n} \in L$ be respectively the upper and lower boundary. Then a boundary event point is detected by verifying:

- if $\phi_{u}, \phi_{v} \in U \cup L$, then $\mathbf{q}$ is in:

- type a, if $\left(\phi_{u}=\phi_{1}\right.$ and $\left.\phi_{v} \neq \phi_{2}\right)$ or $\left(\phi_{v}=\phi_{1}\right.$ and $\left.\phi_{u} \neq \phi_{2}\right)$;

- type b, if $\left(\phi_{u}=\phi_{n}\right.$ and $\left.\phi_{v} \neq \phi_{n-1}\right)$ or $\left(\phi_{v}=\phi_{n}\right.$ and $\left.\phi_{u} \neq \phi_{n-1}\right)$;

- otherwise, if either $\phi_{u} \in U \cup L$ or $\phi_{v} \in U \cup L$, then $\mathbf{q}$ is in:

- type c, if $\phi_{u}=\phi_{1}$ and $\phi_{v} \neq \phi_{2}$;

- type d, if $\phi_{u}=\phi_{1}$ and $\phi_{v}=\phi_{2}$;

- type e, if $\phi_{u}=\phi_{n}$ and $\phi_{u} \neq \phi_{n-1}$;

- type f, if $\phi_{u}=\phi_{n}$ and $\phi_{u}=\phi_{n-1}$. 

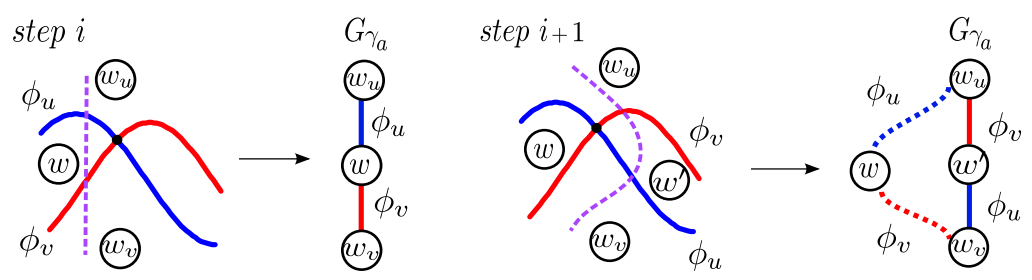

(a)

Figure 14: Updating the graph $G_{\gamma_{a}}$ with respect to the change of the cut $\gamma_{a}$ at an interior event point.

\subsection{Feasible discrete rigid transformation graph construc- tion}

In order to build the FDRT graph in an FRTS $\mathcal{R}$, we use the sweeping algorithm described in Section 4.1. However the cut $\gamma$ in this part sweeps from $\theta_{\min }$ to $\theta_{\max }$ instead of $[0,2 \pi$ [, and contains only the tipping surfaces belonging to $\mathcal{R}$. As described in Section 4.1, an elementary step at each event point consists of (i) updating the graphs $G_{\gamma_{a}}$ and $G_{\gamma_{b}}$ according to the change of $\gamma_{a}$ and $\gamma_{b}$ respectively (explained in the following) and (ii) building the partial graph $\delta G$ from $G_{\gamma_{a}}$ and $G_{\gamma_{b}}$ (see Definition 10). In other words, Step (ii) is done, and we now explain how to perform Step (i). We only describe the procedure for handling event points for simple cases; the degenerate cases are obtained by modifying the procedure of this simple case, as detailed in [17].

At each elementary step of an interior event point $\mathbf{q}=\left\{\phi_{u}, \phi_{v}\right\}$ generated by two tipping curves $\phi_{u}, \phi_{v}$, if the cut on the left of $\mathbf{q}$ is $\gamma=\left(\phi_{1}, \ldots, \phi_{u}, \phi_{v}, \ldots, \phi_{n}\right)$, then after $\mathbf{q}$ we have $\gamma^{\prime}=\left(\phi_{1}, \ldots, \phi_{v}, \phi_{u}, \ldots, \phi_{n}\right)$. According to this change of the cut, the associated graph is modified as shown in Fig. 14.

We now explain how to update the cut at boundary event points, first for the types (a) and (b). Without loss of generality, let $\mathbf{q}=\left\{\phi_{u}, \phi_{v}\right\}$ be a boundary event point generated by two tipping curves $\phi_{u}, \phi_{v}$ and $\gamma, \gamma^{\prime}$ be the cuts before and after $\mathbf{q}$ respectively. Assuming $\gamma=\left(\phi_{1}, \phi_{2}, \ldots, \phi_{n-1}, \phi_{n}\right)$, if $\mathbf{q}$ is on

- the upper boundary, i.e., $\phi_{u}=\phi_{1}$ and $\phi_{v} \neq \phi_{2}$, then $\gamma^{\prime}=\left(\phi_{v}, \phi_{2}, \ldots, \phi_{n-1}, \phi_{n}\right)$;

- the lower boundary, i.e., $\phi_{u}=\phi_{n}$ and $\phi_{v} \neq \phi_{n-1}$, then $\gamma^{\prime}=\left(\phi_{2}, \phi_{3}, \ldots, \phi_{n-1}, \phi_{v}\right)$.

Similarly, the procedures for updating the cut for (c) and (d) are given as follows. Let $\mathbf{q}=\left\{\phi_{u}, \phi_{v}\right\}$ be an event point on the upper boundary, i.e., $\phi_{u}=\phi_{1}$. We have two cases:

- when $\phi_{v}$ goes into $\mathcal{R}_{\mathcal{H}}$, i.e., $\phi_{v} \neq \phi_{2}$, then $\gamma^{\prime}=\left(\phi_{1}, \phi_{v}, \phi_{2}, \ldots, \phi_{n}\right)$;

- when the curve $\phi_{v}$ goes out of $\mathcal{R}_{\mathcal{H}}$, i.e., $\phi_{v}=\phi_{2}$, then $\gamma^{\prime}=\left(\phi_{1}, \phi_{3}, \ldots, \phi_{n}\right)$.

The procedures for (e) and (f) can be considered in the same way. Fig. 15 illustrates the elementary steps for those boundary event points. 

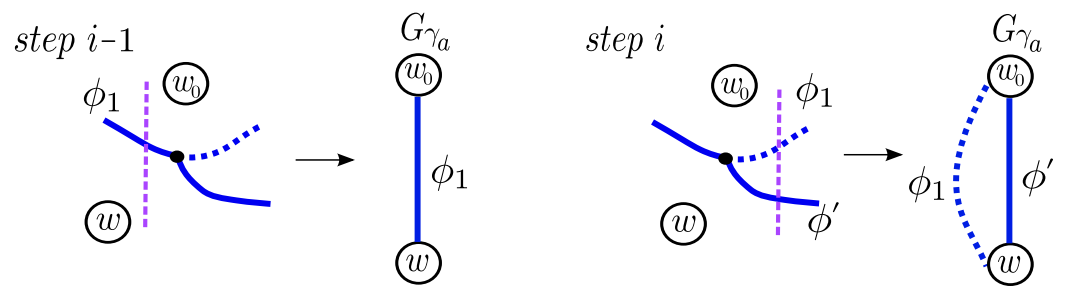

(a)
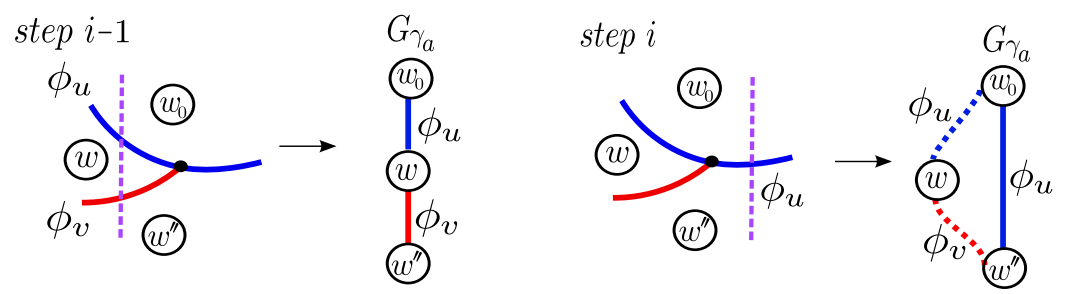

(b)
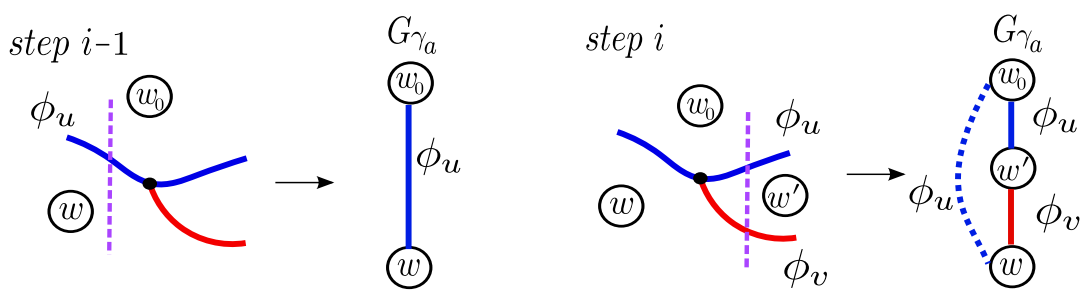

(c)
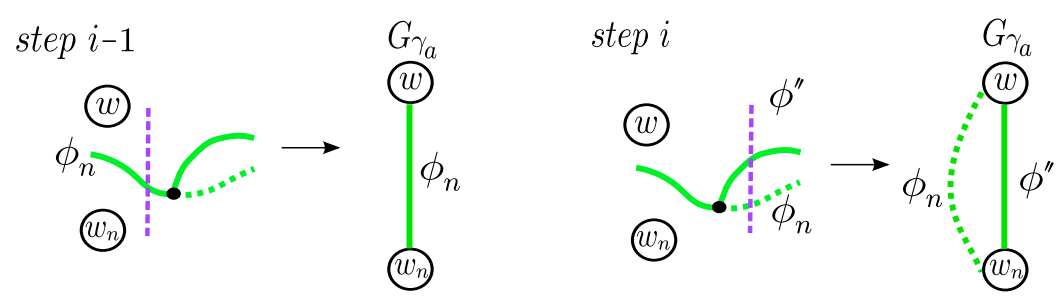

(d)

Figure 15: Illustrations of elementary steps -update $\gamma$ and generate its graph $G_{\gamma}$ - for a tipping curve changing (a) an upper or (b) a lower boundary, and going (c) in or (d) out of an upper boundary.

\section{Complexity analysis}

\subsection{Space complexity of feasible discrete rigid transforma- tion graphs}

\subsubsection{Theoretical results}

The space complexity of an FDRT graph corresponds to the numbers of its vertices and edges. These values directly depend on the number of event points 
involved in its construction, and the number of vertices generated at each event point. It was shown in [17, Proposition 3] that the number of edge is in the same order as the number of vertices. The following discussions, dealing only with vertices, then provide results for the space complexity of FDRT graphs.

As mentioned in Section 4.1, the construction of a DRT graph $G$ is obtained from its projections on the planes $(a, \theta)$ and $(b, \theta)$. In absence of constraints, it was shown in [17] that there are $\mathcal{O}\left(N^{3}\right)$ tipping curves in each plane. Since any two tipping curves intersect in at most two points for $\theta \in[0,2 \pi[$, the number of event points is $\mathcal{O}\left(N^{6}\right)$. Moreover, at each elementary step, i.e., at each event point, the number of generated vertices is $\mathcal{O}\left(N^{3}\right)$. The number of vertices in $G$ is then $\mathcal{O}\left(N^{9}\right)$, and thus this justifies the result already stated in Property 3.

For one pixel-invariance constraint, the complexity analysis scheme remains similar to the non-constrained case. However, some of the DRTs -i.e., some of the vertices of the FDRT graph- become unfeasible, and the number of event points decreases from $\mathcal{O}\left(N^{6}\right)$ to $\mathcal{O}\left(N^{5}\right)$, due to periodicity properties (see [17, Property 4]). Moreover, we derives from [17, Property 5] that the number of tipping curves in the projection on the plane either $(a, \theta)$ or $(b, \theta)$ of the FRTS associated to the given constraint, is decreased from $\mathcal{O}\left(N^{3}\right)$ to $\mathcal{O}\left(N^{2}\right)$. Thus at each elementary step, associated to each event point, the number of generated vertices is $\mathcal{O}\left(N^{2}\right)$. This leads to the following property.

Property 14 The FDRT graph $G$ associated to a digital image of size $N \times N$ under one pixel-invariance constraint has a space complexity $\mathcal{O}\left(N^{7}\right)$.

Geometrically, the associated FRTS correspond to a tube-like volume as illustrated in Fig. 5(c).

For more than one pixel-invariance constraint, the space complexity of the DRT graph does not only depend on the number of constraints, but also on the geometric configuration of the points involved in these constraints. In the worst case, this implies that the space complexity does not necessarily decrease, as intuitively illustrated by the following example.

Let us consider the two pixel-invariance constraints induced by the set of points $\left\{\vec{p}_{1}, \vec{p}_{2}\right\}$ and $\left\{\vec{p}_{1}^{\prime}, \vec{p}_{2}^{\prime}\right\}$, with $\vec{p}_{1}=\vec{p}_{2}+(0,1)$ and $\vec{p}_{1}^{\prime}=\vec{p}_{2}^{\prime}+(0,1)$. While the first constraint reduces the space complexity of $G$ from $\mathcal{O}\left(N^{9}\right)$ to $\mathcal{O}\left(N^{7}\right)$ as stated in Property 14 (see also Fig. 5(c)), the second one reduces the $\theta$ part of the FRTS from $[0,2 \pi[$ to $] 0, \pi[$. Thus with these constraints, the size of $G$ is only divided by a constant factor 2 , and we obtain the following result.

Property 15 The FDRT graph $G$ associated to a digital image of size $N \times N$ under two pixel-invariance constraints has a space complexity of $\mathcal{O}\left(N^{7}\right)$ in the worst case.

However, we show in Section 5.1.2, that the actual space complexities of FDRT graphs under multiple pixel-invariance constraints are generally lower than this worst case. 


\begin{tabular}{|c|r|r|}
\hline \multirow{2}{*}{$N$} & \multicolumn{2}{|c|}{ FDRT Graph } \\
\cline { 2 - 3 } & Vertices & \multicolumn{1}{|c|}{ Edges } \\
\hline 1 & 1 & 0 \\
2 & 147 & 700 \\
3 & 2256 & 11616 \\
4 & 14651 & 78140 \\
5 & 80289 & 430752 \\
6 & 265899 & 1445986 \\
7 & 842137 & 5314904 \\
8 & 2076029 & 13190632 \\
9 & 5103633 & 32291768 \\
10 & 10244909 & 65204024 \\
\hline
\end{tabular}

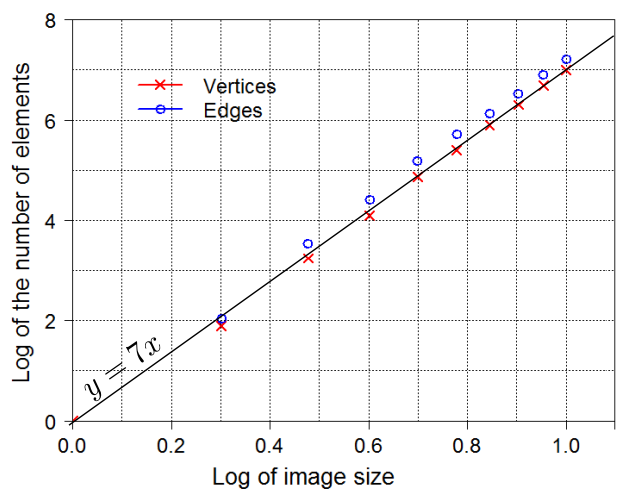

Figure 16: Space complexities of FDRT graphs expressed by the numbers of vertices and edges in FDRT graphs under one pixel-invariance constraint, for images of size $N \times N$.

\subsubsection{Experimental results}

We now describe some experiments and results obtained with the proposed algorithm for FDRT graph construction under pixel-invariance constraints as explained in the previous section. This algorithm was implemented in $\mathrm{C}++$. Experiments were carried out on a personal computer equipped with a $3.0 \mathrm{GHz}$

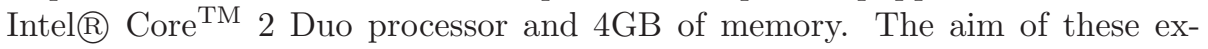
periments is to validate the proposed algorithm with respect to the theoretical complexity results established above, but also to investigate practical complexities for tighter constraints.

The first experiments, illustrated in Fig. 16, deal with FDRT graphs for one pixel-invariance constraint. They confirm the theoretical results established in Property 14. One may notice that in previous works dealing with discrete rotations $[2,26]$, a complexity of only $\mathcal{O}\left(N^{3}\right)$ was established. In these works, no translation was considered. In the current case, we allow translations due to our pixel-invariance formulation. As a result, for a given constraint, we obtain a set of feasible transformations forming a tube which contains arbitrary translations and rotations centered anywhere within a pixel, as illustrated in Fig. 5(c).

As discussed in Section 5.1.1, the space complexity of $G$ under two pixelinvariance constraints is also $\mathcal{O}\left(N^{7}\right)$ (see Property 15) if we consider a pair of pixels separated by a distance of 1 . This constitutes an extreme case. In practice, the complexity of a FDRT graph is generally lower, since the distance between two constraint points is likely to exceed 1 (see Fig. 19(a)). It is reasonable to infer that the longer the distance between these points, the more constrained the feasible transformations, and therefore the lower the complexity of $G$. Following this intuition, we include a distance parameter in the space complexity of $G$. The theoretical complexity of $G$ under two pixel-invariance constraints remains an open problem; however, we propose the following conjec- 

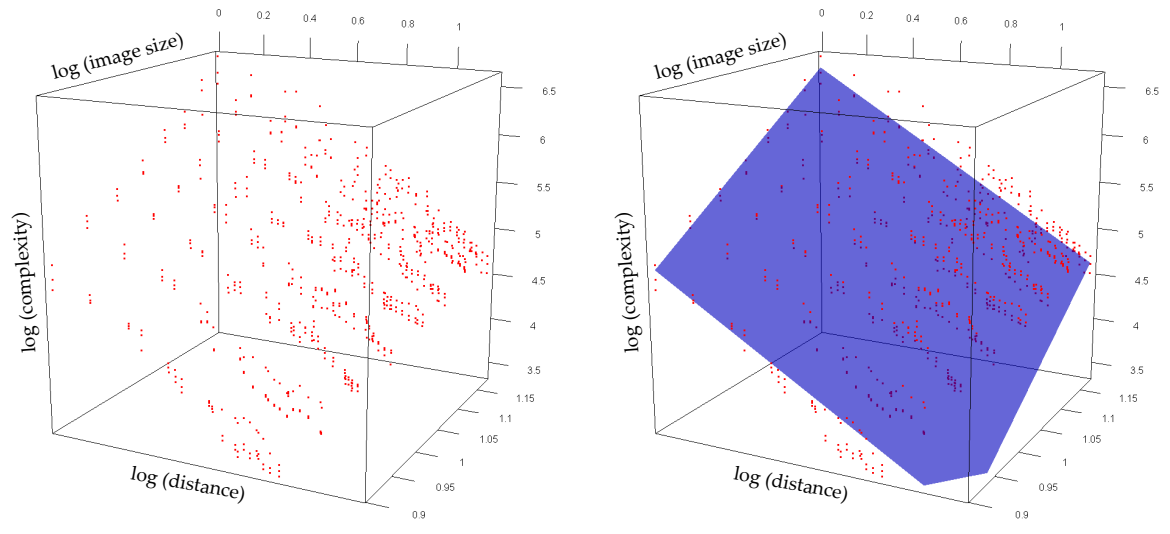

Figure 17: Experimental space complexities of FDRT graphs in the two pixelinvariance constraints case. The complexities are measured over 676 experiments as a function of image size, varying from $8 \times 8$ to $15 \times 15$, and varying distances between two given constraints limited by the image sizes. Left: 3D log plot of distance, image size and space complexity. Right: least-squares best plane fit (colored in blue).

ture which links the complexity of $G$ with the distance between points of given constraints.

Conjecture 16 The FDRT graph $G$ associated to a digital image of size $N \times N$ under two pixel-invariance constraints has a space complexity of $\mathcal{O}\left(N^{\alpha} d^{-\beta}\right)$, with $\alpha, \beta>0$, where $d$ denotes the Euclidean distance between two pixel-invariance constraints.

The $\alpha$ and $\beta$ values are assumed to be constant, and we propose to estimate their values experimentally. Some results are given in Fig. 17 and 19(a) to verify the proposed conjecture. Experiments were carried out by using images of sizes varying from $8 \times 8$ to $15 \times 15$. For each image size, we considered a sample of several distances, and we randomly chose several two pixel-invariance constraints for each distance. The estimation of $\alpha$ and $\beta$ in $\mathcal{O}\left(N^{\alpha} d^{-\beta}\right)$ can be interpreted as a plane fitting estimation in the 3D space induced by $N, d$ and the space complexity. By using the least-squares method, we obtained $\alpha=5.5$ and $\beta=1.6$ with a residual standard error for derived parameters of 0.1244 on 676 experiments performed and the adjusted multiple correlation coefficient of 0.9993, that corresponds to a very good fit.

We now consider more than two constraints. We may have expected the FDRT graph $G$ associated to these constraints to be reduced. Nevertheless, this is not always true. Indeed, the space complexity of $G$ then depends on the geometric configuration of the pixel-invariance constraints, as illustrated by the following examples. Let us consider two pixel-invariance constraints (in red and blue in Fig. 18(a)). When a supplementary constraint is added (for instance 


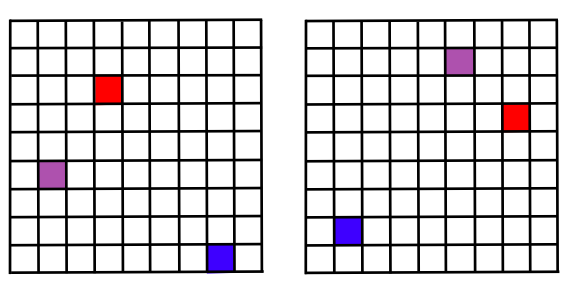

(a)

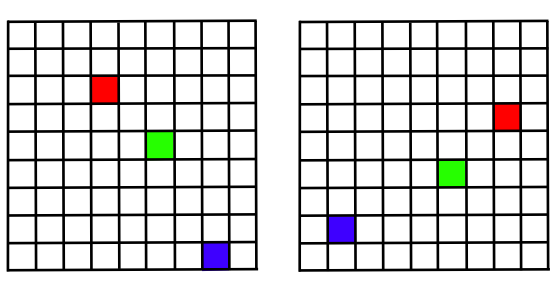

(b)

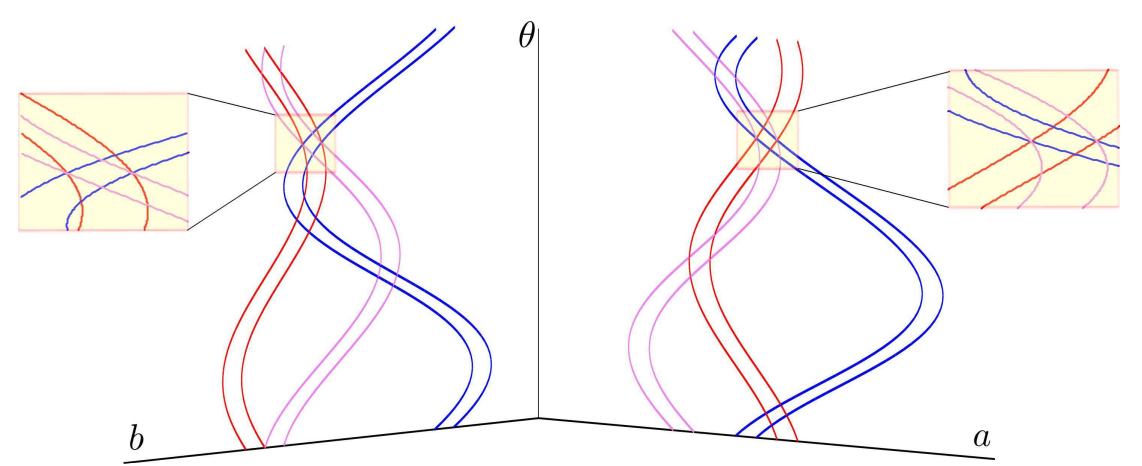

(c)

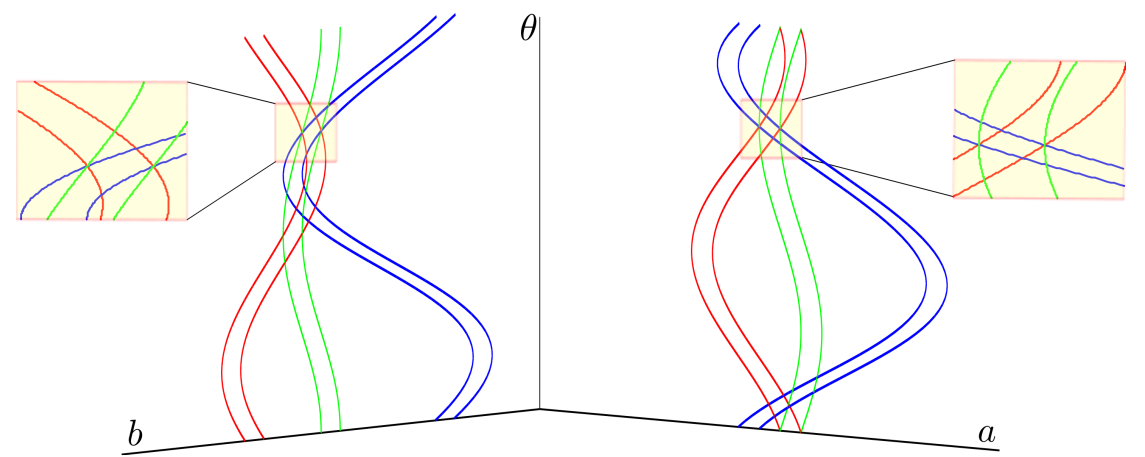

(d)

Figure 18: For two given pixel-invariance constraints (in red and blue), there exists a supplementary pixel-invariance constraint (in purple) that contributes to reducing the associated FRTS (a). There also exists a supplementary pixelinvariance constraint (in green) that does not (b). (c) and (d) illustrate the cross-sections of the FRTS, on the planes $(a, \theta)$ and $(b, \theta)$, induced by the constraints given in (a) and (b) respectively.

the purple one in Fig. 18(a)) we can see in Fig. 18(c) that the FRTS is strictly reduced, and so is the FDRT graph $G$. On the contrary, let us consider the supplementary constraint determined by green pixels in Fig. 18(b). We can observe that such a constraint does not reduce the FRTS, and the FDRT graph 


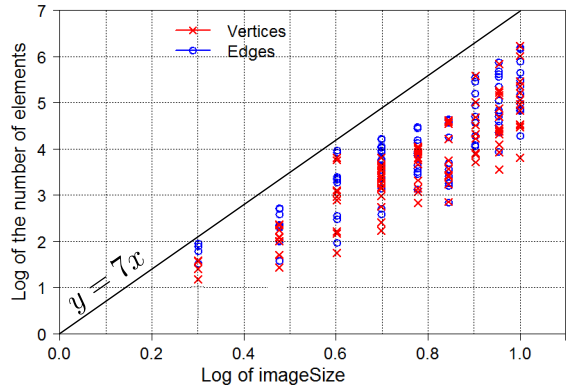

(a)

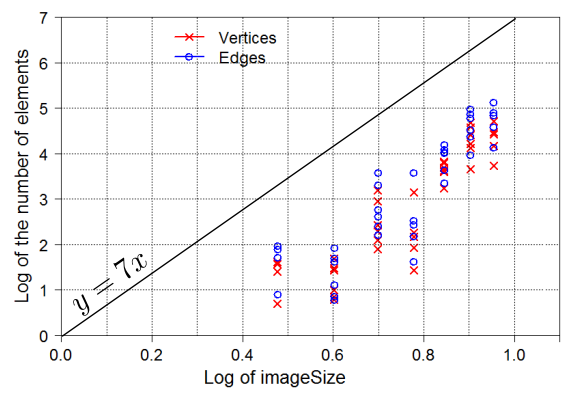

(c)

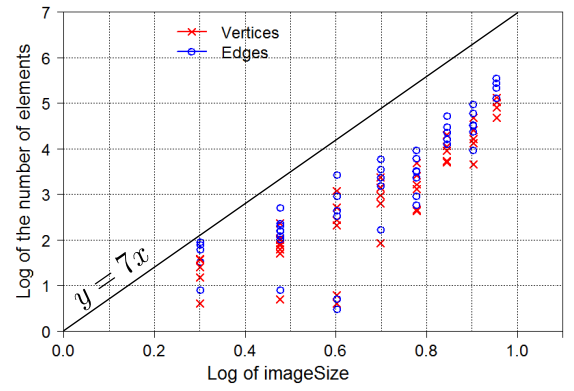

(b)

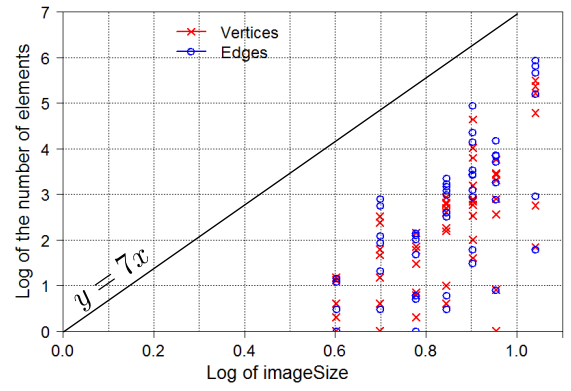

(d)

Figure 19: Experimental results of space complexities of FDRT graphs for two (a), three (b), five (c) and ten (d) pixel-invariance constraints.

$G$ then remain unchanged (see Fig. 18(d)).

However, in practice, the higher the number of constraints, the lower the complexity of the FDRT. This is illustrated in Figure 19(b-d) that corresponds to experiments for 3,5 and 10 random pixel-invariance constraints, respectively.

\subsection{Time complexity of feasible discrete rigid transforma- tion graph construction}

From Section 4, we know that the construction of an FDRT graph is obtained from its projections on the planes $(a, \theta)$ and $(b, \theta)$, and can be performed in three successive steps:

1. finding the boundary of the FRTS $\mathcal{R}$;

2. finding the event points in $\mathcal{R}$, more precisely, in the projections $\mathcal{R}_{\mathcal{H}}$ and $\mathcal{R}_{\mathcal{V}}$ of $\mathcal{R}$ on the planes $(a, \theta)$ and $(b, \theta)$ respectively; and

3. building the FDRT graph $G$ associated to $\mathcal{R}$.

In each of the $(a, \theta)$ and $(b, \theta)$ planes, the event points are generated by $2 m$ tipping curves, induced by the $m$ given constraints (with $0 \leq m \leq N^{2}$ ). 
Moreover, it is proved in [17] that the number of event points for $m$ tipping curves is $\mathcal{O}\left(\mathrm{m}^{2}\right)$. In Step 1, the verification of the cut separation is done in $\mathcal{O}(m)$ for every point that has to be sorted. This sorting and verification lead to a complexity of $\mathcal{O}\left(m^{2} \log m\right)$ and $\mathcal{O}\left(\mathrm{m}^{3}\right)$ respectively. Therefore, Step 1 has a time complexity of $\mathcal{O}\left(\mathrm{m}^{3}\right)$, which is equivalent to $\mathcal{O}\left(N^{6}\right)$.

In Step 2, the process of detecting whether a tipping surface passes through $\mathcal{R}$ can be done in linear time with respect to the size of the boundary of $\mathcal{R}$, i.e., $\mathcal{O}(m)$. Since there are $\mathcal{O}\left(N^{3}\right)$ tipping surfaces, the time complexity of this sub-step is $\mathcal{O}\left(m N^{3}\right)$. Then, we search the event points in $\mathcal{R}$. Due to the periodicity of tipping curves [17, Property 4], we know that the total number of event points in $\mathcal{R}$ is $\mathcal{O}\left(N^{5}\right)$, and thus the mandatory sorting of these event points has a time complexity of $\mathcal{O}\left(N^{5} \log N\right)$.

The sweep of a cut, in Step 3, requires $\mathcal{O}\left(N^{5}\right)$ iterations (one for each event point), and at each iteration, $\mathcal{O}\left(N^{2}\right)$ vertices are generated. Therefore, Step 3 has a time complexity of $\mathcal{O}\left(N^{7}\right)$, which is the most costly step in the algorithm.

Finally, the FDRT graph $G$ for a given image of size $N \times N$ under $m$ constraints is then constructed with a time cost of $\mathcal{O}\left(N^{7}\right)$.

\section{Conclusion}

This article continued the study initiated in $[16,17]$ by investigating the effects of geometric constraints on rigid transformations, applied to digital images. By enforcing the correspondence between one or several pairs of pixels, we restricted allowable transformations to a parameter subspace, called a feasible rigid transformation set (FRTS), in which all such constraints are satisfied. A proposed algorithm allowed us to build a combinatorial structure (namely a graph) for modeling the subdivision of the FRTS on a subset of $\mathbb{Z}^{2}$ of size $N \times N$. We theoretically analysed the complexity of this graph with one given pixel-invariance constraint to be $\mathcal{O}\left(N^{7}\right)$. For two constraints, the complexity could not be theoretically given. However, we experimentally evaluated its complexity as $\mathcal{O}\left(N^{5.5} d^{-1.6}\right)$, where $d$ denotes the Euclidean distance between two pixel-invariance constraints.

From the discussion of Section 5.1.2 regarding the complexity of the FDRT graph under more than two constraints, it appears that the pixels can be classified into two categories: those which can be involved in supplementary constraints that reduce the FRTS, and those that cannot (see Fig. 20). The later ones are called static pixels. Based on this classification, it may be possible to choose only those which actually reduce the FRTS when iteratively defining constraints. This strategy may be investigated in further works.

\section{References}

[1] Amintoosi, M., Fathy, M., Mozayani, N.: A fast image registration approach based on SIFT key-points applied to super-resolution. Imaging 


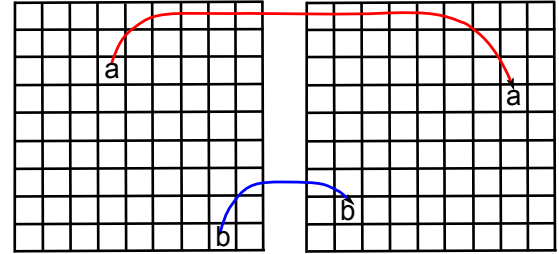

(a) Two pixel-invariance constraints.

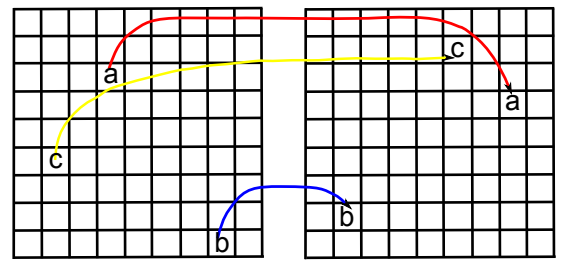

(c) Three pixel-invariance constraints.

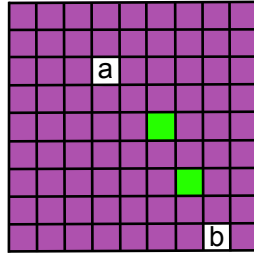

(b) Static vs. nonstatic pixels pixels in (a).

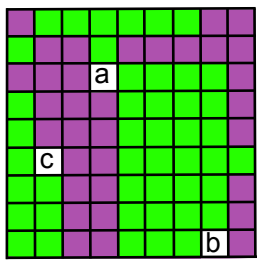

(d) Static vs. nonstatic pixels pixels in (c).

Figure 20: (a,c) Pixel-invariance constraints (denoted by the arrows). (b,d) Classification of the remainder pixels in the image under constraints given in $(\mathrm{a}, \mathrm{c})$ respectively. The green pixels are static and the purple ones are not (see text).

Science Journal 60(4), 185-201 (2011)

[2] Amir, A., Kapah, O., Tsur, D.: Faster two-dimensional pattern matching with rotations. Theoretical Computer Science 368(3), 196-204 (2006)

[3] Andres, E.: The quasi-shear rotation. In: DGCI, Proceedings, Lecture Notes in Computer Science, vol. 1176, pp. 307-314. Springer (1996)

[4] Bishop, C.M.: Pattern Recognition and Machine Learning. Springer-Verlag New York, Inc. (2006)

[5] Chan, T.M.: On levels in arrangements of surfaces in three dimensions. In: SODA, Proceedings, pp. 232-240. ACM-SIAM (2005)

[6] Coeurjolly, D., Blot, V., Jacob-Da Col, M.A.: Quasi-affine transformation in 3-D: Theory and algorithms. In: IWCIA, Proceedings, Lecture Notes in Computer Science, vol. 5852, pp. 68-81. Springer (2009)

[7] Edelsbrunner, H., Guibas, L.J.: Topologically sweeping an arrangement. In: STOC, Proceedings, pp. 389-403. ACM (1986)

[8] Gose, E., Johnsonbaugh, R., Jost, S.: Pattern Recognition and Image Analysis. Prentice-Hall, Inc. (1996) 
[9] Gribaa, N., Noblet, V., Khalifa, N., Faisan, S., Hamrouni, K.: Binary image registration based on geometric moments: Application to the registraion of 3D segmented CT head images. International Journal of Image and Graphics 12(2) (2012)

[10] Harris, C.: Tracking with rigid models. In: A. Blake, A. Yuille (eds.) Active Vision, pp. 59-73. MIT Press (1993)

[11] Hundt, C., Liśkiewicz, M.: On the complexity of affine image matching. In: STACS, Proceedings, Lecture Notes in Computer Science, vol. 4393, pp. 284-295. Springer (2007)

[12] Hundt, C., Liśkiewicz, M.: Combinatorial bounds and algorithmic aspects of image matching under projective transformations. In: MFCS, Proceedings, Lecture Notes in Computer Science, vol. 5162, pp. 395-406. Springer (2008)

[13] Hundt, C., Liśkiewicz, M., Ragnar, N.: A combinatorial geometrical approach to two-dimensional robust pattern matching with scaling and rotation. Theoretical Computer Science 410(51), 5317-5333 (2009)

[14] Jacob, M.A., Andres, E.: On discrete rotations. In: DGCI, Proceedings, pp. 161-174 (1995)

[15] Maintz, J., Viergever, M.: A survey of medical image registration. Medical Image Analysis 2(1), 1-36 (1998)

[16] Ngo, P., Kenmochi, Y., Passat, N., Talbot, H.: Combinatorial properties of $2 \mathrm{D}$ discrete rigid transformations under pixel-invariance constraints. In: IWCIA, Proceedings, Lecture Notes in Computer Science, vol. 7655, pp. 234-248. Springer (2012)

[17] Ngo, P., Kenmochi, Y., Passat, N., Talbot, H.: Combinatorial structure of rigid transformations in $2 \mathrm{D}$ digital images. Computer Vision and Image Understanding 117(4), 393-408 (2013)

[18] Ngo, P., Kenmochi, Y., Passat, N., Talbot, H.: Sufficient conditions for topological invariance of $2 \mathrm{D}$ digital images under rigid transformations. In: DGCI, Proceedings, Lecture Notes in Computer Science, vol. 7749, pp. 155-168. Springer (2013)

[19] Nouvel, B., Rémila, E.: Configurations induced by discrete rotations: Periodicity and quasi-periodicity properties. Discrete Applied Mathematics $147(2-3), 325-343$ (2005)

[20] Nouvel, B., Rémila, E.: Incremental and transitive discrete rotations. In: IWCIA, Proceedings, Lecture Notes in Computer Science, vol. 4040, pp. 199-213. Springer (2006) 
[21] Pennec, X., Ayache, N., Thirion, J.P.: Landmark-based registration using features identified through differential geometry. In: I.N. Bankman (ed.) Handbook of Medical Imaging, chap. 31, pp. 499-513. Academic Press (2000)

[22] Reveillès, J.P.: Géométrie discrète, calcul en nombres entiers et algorithmique. Thèse d'état, Université Strasbourg 1 (1991)

[23] Richman, M.S.: Understanding discrete rotations. In: ICASSP, Proceedings, vol. 3, pp. 2057-2060. IEEE (1997)

[24] Rosen, K.H.: Elementary Number Theory and its Applications, 3rd edn. Addison-Wesley (1992)

[25] Sharir, M.: Recent developments in the theory of arrangements of surfaces. In: FSTTCS, Proceedings, Lecture Notes in Computer Science, vol. 1738, pp. 1-21. Springer (1999)

[26] Thibault, Y.: Rotations in 2D and 3D discrete spaces. Ph.D. thesis, Université Paris-Est (2010)

[27] Yilmaz, A., Javed, O., Shah, M.: Object tracking: A survey. ACM Computing Surveys 38(4), 1-45 (2006)

[28] Zitová, B., Flusser, J.: Image registration methods: A survey. Image and Vision Computing 21(11), 977-1000 (2003) 